\title{
Modeling Schistosomiasis and HIV/AIDS Codynamics
}

\author{
S. Mushayabasa ${ }^{1}$ and C. P. Bhunu ${ }^{1,2}$ \\ ${ }^{1}$ Modelling Biomedical Systems Research Group, Department of Applied Mathematics, National University of Science and Technology, \\ P.O. Box 939 Ascot, Bulawayo, Zimbabwe \\ ${ }^{2}$ Department of Veterinary Medicine, University of Cambridge, Cambridge CB3 OES, UK
}

Correspondence should be addressed to S. Mushayabasa, steadymushaya@gmail.com

Received 1 March 2010; Accepted 11 September 2010

Academic Editor: Sivabal Sivaloganathan

Copyright ( $) 2011$ S. Mushayabasa and C. P. Bhunu. This is an open access article distributed under the Creative Commons Attribution License, which permits unrestricted use, distribution, and reproduction in any medium, provided the original work is properly cited.

\begin{abstract}
We formulate a mathematical model for the cointeraction of schistosomiasis and HIV/AIDS in order to assess their synergistic relationship in the presence of therapeutic measures. Comprehensive mathematical techniques are used to analyze the model steady states. The disease-free equilibrium is shown to be locally asymptotically stable when the associated disease threshold parameter known as the basic reproduction number for the model is less than unity. Centre manifold theory is used to show that the schistosomiasis-only and HIV/AIDS-only endemic equilibria are locally asymptotically stable when the associated reproduction numbers are greater than unity. The impact of schistosomiasis and its treatment on the dynamics of HIV/AIDS is also investigated. To illustrate the analytical results, numerical simulations using a set of reasonable parameter values are provided, and the results suggest that schistosomiasis treatment will always have a positive impact on the control of HIV/AIDS.
\end{abstract}

\section{Introduction}

Schistosomiasis, also known as bilharzia after Theodor Bilharz who first identified the parasite in Egypt in 1851, is a disease caused by blood flukes [1]. It affects millions of people worldwide, especially in South America, the Middle East, and Southeast Asia where it remains a public health problem and poses a threat to 600 million people in more than 76 countries [1]. The disease is often associated with water resource development projects, such as dams and irrigation schemes, where the snail intermediate hosts of the parasite breed [2]. Human schistosomiasis (which has a relatively low mortality rate, but a high morbidity rate) is a family of diseases primarily caused by three species of the genus Schistosoma or flat worms. The adult worms inhabit the blood vessels lining either the intestine or bladder, depending on the species of the worm [3]. The highest number of human schistosomiasis infections is caused by $S$. haematobium, which has a predilection for the blood vessels around the bladder and causes urinary disease [4]. Schistosomiasis is the second most prevalent neglected tropical diseases after hookworm (192 million cases), accounting for $93 \%$ of the world's number of cases and possibly associated with increased horizontal transmission of HIV/AIDS [5].
On the other hand, the number of people living with HIV worldwide continued to grow in 2008, reaching an estimated 33.4 million, which is more than $20 \%$ higher than the number in 2000, and the prevalence was roughly threefold higher than in 1990 [6]. The HIV virus, by holding the immune system hostage, has opened many gates for pathological interactions with other diseases [7]. Schistosomiasis and HIV infections have major effects on the host immune response, and coinfection (of the two diseases which may increase the complexity of treatment for people living with HIV and may contribute to poorer medical outcomes) is widespread [8]. While schistosomiasis infections are caused by diverse species from three phyla, HIV is essentially a single entity. There is some evidence that schistosomiasis infection provides some benefit in some instances like the atopic disease $[9,10]$, and the inflammatory pathology of autoimmune disease [11-13]. For bacterial and viral infections, impaired control of replication and elimination may lead to a detrimental outcome [14-17]. That HIV infection is detrimental to the immune response to many pathogens is quite clear and poor regulation of immune system in advanced HIV infection is illustrated by an increased incidence of hypersensitive drug reactions $[18,19]$. Studies that examine the codynamics of HIV and schistosomiasis infections have shown a significant 
association between HIV and the presence of S. haematobium eggs in the genital samples, supporting the argument that schistosomiasis infection enhances HIV susceptibility when genital lesions are present [20]. Host-parasite interactions such as schistosomiasis, where inflammatory responses have persisted through evolution, perhaps due to selective advantage for parasite egg excretion, may be more detrimental with regard to HIV infection [21].

Although the negative impact of the synergetic interactions between HIV and schistosomiasis has shown to be a public health burden, only few statistical or mathematical models have been used to explore the consequences of their joint dynamics at the population level. There are plenty of single disease dynamic models. A significant number focus on HIV/AIDS [22-26] or on the transmission dynamics of schistosomiasis [27-37]. Schistosomiasis model (24) considered in this study differs from those found in the literature in that we consider Schistosoma mansoni a human blood fluke which causes schistosomiasis and is the most widespread and the fresh water snail Biomphalaria glabrata serves as the main intermediate host, while the HIV/AIDS model (7) is an extension of the model by Murray [38] by including HIV therapy while neglecting the issue of seropositivity considered in [38]. Mathematical modeling assessing the impact of schistosomiasis on the transmission dynamics of HIV/AIDS is rare [39].

Quantifying by how much treatment of schistosomiasis affects HIV/AIDS dynamics will require an extensive sensitivity analysis with parameter values estimated from real and recent coinfection data. Nevertheless, this theoretical study provides a framework for the potential benefit of schistosomiasis treatment on the dynamics of HIV and highlights the fact that global public health challenges require comprehensive and multipronged approaches to dealing with coinfections [7], and current intervention efforts that focus on a single infection at a time may be losing substantial rewards of dealing synergistically and concurrently with multiple infectious diseases in one host. To the best of our knowledge, except for the study in [39] where the cointeraction of schistosomiasis and HIV without any form of treatment is investigated, this work is possibly the first to give a theoretical mathematical account of the impact of schistosomiasis on HIV dynamics in the presence of both schistosomiasis treatment and antiretroviral therapy at the population level.

The rest of the paper is structured as follows. In the next section, we present the schistosomiasis and HIV/AIDS coinfection model. In Section 3 we determine sufficient conditions for local stability of the disease-free and endemic equilibria and analyze the reproduction number for the two diseases separately while Section 4 provides a comprehensive analysis of the full model. Section 5 provides numerical results while Section 6 concludes the paper.

\section{Model Description}

The proposed model is an extension of an earlier study [39], which did not account for any intervention strategy. The schistosomiasis and HIV models will be coupled via the force of infection, and in the absence of any of the diseases (hence no coinfection), the two basic disease submodels can be decoupled from the general model (see Sections 3.1.4 and 3.3.1). The population of interest is divided into several compartments dictated by the epidemiological stages (disease status), namely, susceptibles $S_{H}(t)$, who are not yet infected by either HIV or schistosomiasis, schistosomiasisinfected individuals $I_{B}(t)$, HIV-infected individuals not yet displaying symptoms of AIDS $I_{H}(t)$, individuals infected with HIV showing symptoms of AIDS $A_{H}(t)$, individuals dually infected with schistosomiasis and HIV displaying symptoms of schistosomiasis only $I_{H_{B}}(t)$, individuals dually infected with schistosomiasis and HIV displaying symptoms of schistosomiasis and AIDS $A_{H_{B}}(t)$, treated individuals infected with HIV only, showing symptoms of AIDS $A_{\mathrm{HT}_{A}}(t)$, and treated individuals dually infected with schistosomiasis and HIV displaying symptoms of schistosomiasis and AIDS $A_{H_{T_{B}}}(t)$. Other important populations to consider in this model are the susceptible snails $S_{s}(t)$, infected snails $I_{s}(t)$, miracidia population $M(t)$, and the cercariae population $P(t)$. Individuals move from one class to the next as the disease progresses and/or through dual infection. We further make the following assumptions for the model.

(i) There is no vertical transmission of both infections in humans.

(ii) Infected snails do not reproduce due to castration by miracidia.

(iii) Seasonal and weather variations do not affect snail populations and contact patterns.

(iv) Susceptible humans become infected with schistosomiasis only through contact with free-living pathogen in infested waters.

At any time, new recruits enter the human and snail populations through birth/migration at constant rates $\Lambda_{H}$ and $\Lambda_{S}$, respectively. There is a constant natural death rate $\mu_{H}$ in each human subclass. The force of infection associated with HIV infection, denoted by $\lambda_{H}$, is given by

$$
\lambda_{H}(t)=\frac{\beta_{H} c\left[I_{H}+I_{H_{B}}+\eta\left(A_{H}+A_{H_{B}}\right)+\kappa\left(A_{H_{T_{A}}}+A_{H_{T_{B}}}\right)\right]}{N_{H}},
$$

with $\beta_{H}$ being the probability of HIV transmission per sexual contact, $c$ is the effective contact rate for HIV infection to occur, and $\eta>1$ models the fact that individuals in the AIDS stage and not on antiretroviral therapy are more infectious since the viral load is correlated with infectiousness [42]. It is assumed that individuals on antiretroviral therapy transmit infection at the smallest rate $\kappa$ (with $0<\kappa<1$ ) because of the fact that these individuals have very small viral load. It has been estimated by an analysis of longitudinal cohort data that antiretroviral therapy reduces per-partnership infectivity by as much as $60 \%$ (so that $\kappa=0.4$ ) [41]. Thus, the total human 
population $N_{H}(t)$ is given by

$$
\begin{aligned}
N_{H}(t)= & S_{H}(t)+I_{H}(t)+A_{H}(t)+A_{H_{T_{A}}}(t) \\
& +I_{\mathrm{B}}(t)+I_{H_{B}}(t)+A_{H_{B}}(t)+A_{H_{T_{B}}}(t) .
\end{aligned}
$$

Susceptible individuals acquire schistosomiasis following infection at a rate $\lambda_{P}$, where

$$
\lambda_{P}=\frac{\beta_{P} P(t)}{P_{0}+\epsilon P(t)},
$$

with $\beta_{P}$ being the maximum rate of exposure, $\epsilon$ is the limitation of the growth velocity of cercariae with the increase of cases, and $P_{0}$ is the half saturation constant. In the absence of the parasite, the functional response of individuals susceptible to the pathogen (schistosomiasis) is given by $\left(\lambda_{P} / \beta_{P}\right)\left[S_{H}(t)+I_{H}(t)+A_{H}(t)+A_{H_{T_{A}}}(t)\right]$, a modified Holling's type-II functional response (also known as the Michaelis-Menten function when $\epsilon=1$ ), the response refers to the change in the density of susceptibles per unit time per pathogen as the schistosomiasis susceptible population density changes. From the functional response, we note that at low parasite density, contacts are directly proportional to host density, but a maximum rate of contact is reached at very high densities (saturation incidence). Individuals infected with schistosomiasis have an additional diseaseinduced death rate $d_{B}$. Similarly, susceptible and infected snails have a natural death rate $\mu_{S}$, and the infected snails have an additional disease-induced death rate $d_{S}$. The total snail population is given by $N_{S}(t)=S_{S}(t)+I_{S}(t)$.

Considering a schistosomiasis-infected individual, a number (portion) $N_{E}$ of eggs leave the body through excretion (faeces and urine) and find their way into the fresh water supply where they hatch into free swimming ciliated miracidium at a rate $\gamma$ for individuals without AIDS. Given the weakened immune system of AIDS individuals, they tend to excrete more often, thus releasing more eggs which will hatch into miracidia at a rate $\sigma \gamma, \sigma>1$. If the miracidium reaches a fresh water with snails of a suitable species, it penetrates at a rate $\lambda_{M}$, where

$$
\lambda_{M}=\frac{\beta_{M} M(t)}{M_{0}+\epsilon M(t)},
$$

and transforms into a sporocyst otherwise, the miracidia die naturally at a rate $\mu_{M}$. The infected snails release a second form of free swimming larva called a cercariae which is capable of infecting humans at rate $\theta$. Some cercariae also die naturally at a rate $\mu_{P}$. Individuals infected with schistosomiasis are infected with HIV at a rate $\delta \lambda_{H}$ with $\delta>$ 1 since infection by schistosomiasis creates wounds within the urethra as eggs are being released, which increases the likelihood of HIV infection per sexual contact. Individuals with HIV progress to the AIDS stage at a rate $\rho$. Individuals in the AIDS stage have an additional disease-induced death rate $d_{A}$. We assume that antiretroviral therapy is given to AIDS individuals who are ill and have experienced AIDS-defining symptoms, or whose CD4+ T cell count is below $200 / \mu \mathrm{L}$, which is the recommended AIDS defining stage [42]. Thus,
AIDS patients are assumed to get antiretroviral therapy at a constant rate $\alpha$. Treated AIDS patients eventually succumb to AIDS-induced mortality at a reduced rate modeled by the parameter $\tau(0<\tau<1)$. Individuals treated for schistosomiasis are assumed to recover at a constant rate $\omega$, and $\omega_{1}$ denotes AIDS patients who have recovered from schistosomiasis but are on antiretroviral therapy since the latter is a life treatment. The model flowchart for the interaction of the two diseases is shown in Figure 1 and parameters described will assume values in Table 1.

From the aforementioned model description and assumptions, we establish the following deterministic system of nonlinear differential equations

$$
\begin{aligned}
& \left\{\begin{array}{l}
\frac{d S_{H}}{d t}=\Lambda_{H}+\omega I_{B}-\left(\lambda_{H}+\lambda_{P}\right) S_{H}-\mu_{H} S_{H} \\
\frac{d I_{B}}{d t}=\lambda_{P} S_{H}-\delta \lambda_{H} I_{B}-\left(\mu_{H}+\omega+d_{B}\right) I_{B}
\end{array}\right. \\
& \frac{d I_{H}}{d t}=\lambda_{H} S_{H}+\omega I_{H_{B}}-\lambda_{P} I_{H}-\left(\mu_{H}+\rho\right) I_{H}, \\
& \frac{d A_{H}}{d t}=\rho I_{H}+\omega A_{H_{B}}-\lambda_{P} A_{H}-\left(\mu_{H}+\alpha+d_{A}\right) A_{H}, \\
& \frac{d A_{H_{T_{A}}}}{d t}=\alpha A_{H}+\omega A_{H_{T_{B}}}-\lambda_{p} A_{H_{T_{A}}} \\
& -\left(\mu_{H}+\tau d_{A}\right) A_{H_{T_{A}}}, \\
& \text { Model system }\left\{\begin{array}{l}
\frac{d I_{H_{B}}}{d t}=\delta \lambda_{H} I_{B}+\lambda_{P} I_{H}-\left(\rho+\omega+\mu_{H}+d_{B}\right) I_{H_{B}}, \\
\frac{d A_{H_{B}}}{d t}=\lambda_{P} A_{H}+\rho I_{H_{B}}-\left(\mu_{H}+\omega+d_{A}+d_{B}\right) A_{H_{B}},
\end{array}\right. \\
& \frac{d A_{H_{T_{B}}}}{d t}=\lambda_{P} A_{H_{T_{A}}}+\alpha A_{H_{B}} \\
& -\left(\mu_{H}+\omega+\tau d_{A}+d_{B}\right) A_{H_{T_{B}}}, \\
& \frac{d M}{d t}=N_{E} \gamma\left(I_{B}+I_{H_{B}}+\sigma A_{H_{B}}+\sigma A_{H_{T_{B}}}\right)-\mu_{M} M, \\
& \frac{d S_{S}}{d t}=\Lambda_{S}-\lambda_{M} S_{S}-\mu_{S} S_{S} \\
& \begin{array}{l}
\frac{d I_{S}}{d t}=\lambda_{M} S_{S}-\left(\mu_{S}+d_{S}\right) I_{S} \\
\frac{d P}{d t}=\theta I_{S}-\mu_{P} P
\end{array}
\end{aligned}
$$

2.1. Model Basic Properties. In this section, we study the basic properties of the solutions of model system (5), which are essential in the proofs of stability.

Lemma 1. The equations preserve positivity of solutions.

Proof. Considering the human population only, the vector field given by the right-hand side of (5) points inward on the boundary of $\mathbb{R}_{+}^{8} \backslash\{0\}$. For example, if $A_{H}=0$, then, $A_{H}^{\prime}=$ $\rho I_{H}+\omega A_{H_{B}} \geq 0$. In an analogous manner, the same result can be shown for the other model components (variables). We shall use the human population to illustrate the boundedness of solutions for model system (5). 


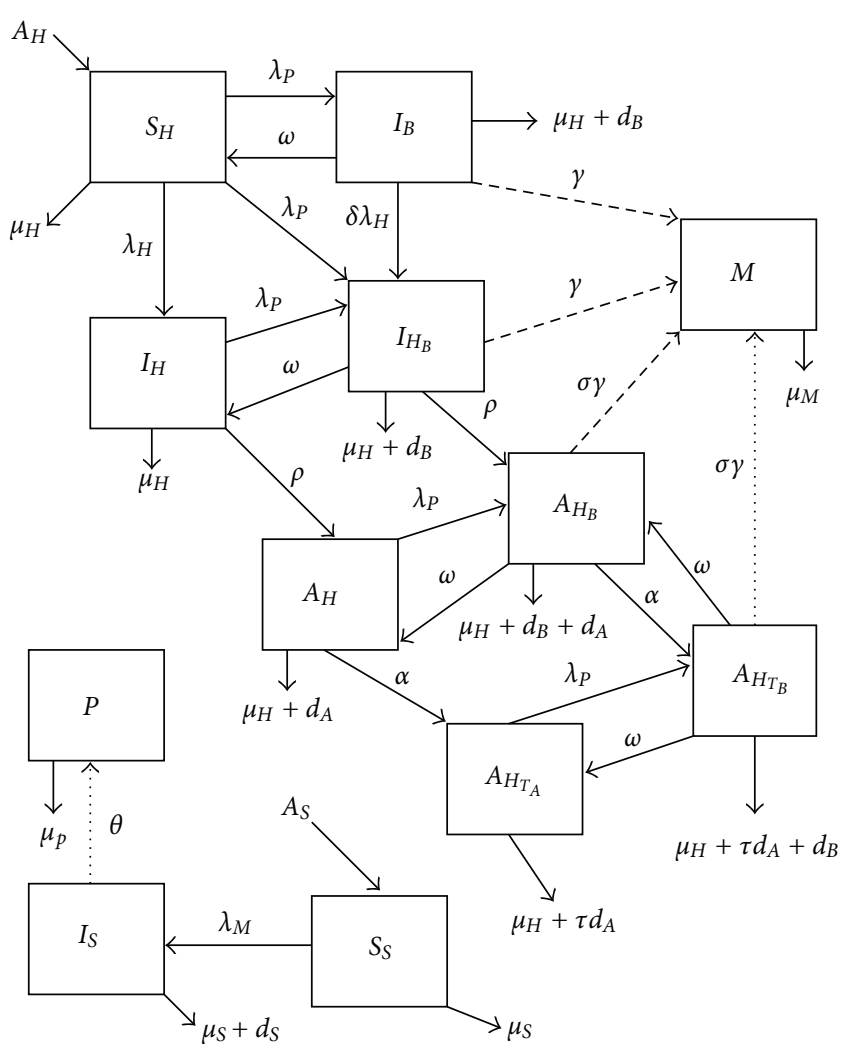

Figure 1: Model flow diagram.

Lemma 2. Each nonnegative solution of model system (5) is bounded in $L^{1}$-norm.

Proof. Consider the human population only, and let $L_{H}^{1} \in$ $L^{1}$; then, the norm $L_{H}^{1}$ of each nonnegative solution in $N_{H}$ is given by $\max \left\{N_{H}(0), \Lambda_{H} / \mu_{H}\right\}$. Thus, the norm $L_{H}^{1}$ satisfies the inequality $N_{H}^{\prime} \leq \Lambda-\mu_{H} N_{H}$. Solutions to the equation $Q^{\prime}=\Lambda-\mu Q$ are monotone increasing and bounded by $\Lambda / \mu$ if $Q(0)<\Lambda / \mu$. They are monotone decreasing and bounded above if $Q(0) \geq \Lambda / \mu$. Since $N_{H}^{\prime} \leq Q^{\prime}$, the claim follows and in a similar fashion, the remaining model variables can be shown to bounded.

Corollary 1. The region

$$
\Phi=\left\{\begin{array}{l}
\left(S_{H}, I_{B}, I_{H}, A_{H}, A_{H_{T_{A}}}, I_{H_{B}}, A_{H_{B}}, A_{H_{T_{B}}}\right) \\
\in \mathbb{R}_{+}^{8}: N_{H} \leq \frac{\Lambda_{H}}{\mu_{H}} \\
M \in \mathbb{R}_{+}: M \leq \frac{\gamma \Lambda_{H} N_{E}(1+\sigma)}{\mu_{M} \mu_{H}} \\
\left(S_{S}, I_{S}\right) \in \mathbb{R}_{+}^{2}: N_{S} \leq \frac{\Lambda_{S}}{\mu_{S}} \\
P \in \mathbb{R}_{+}: P \leq \frac{\theta \Lambda_{S}}{\mu_{P} \mu_{S}}
\end{array}\right.
$$

is invariant and attracting for system (5).
Theorem 1. For every nonzero, nonnegative initial value, solutions of model system (5) exist for all time $t>0$.

Proof. Local existence of solutions follows from standard arguments since the right-hand side of (5) is locally Lipschitz. Global existence follows from the a priori bounds.

\section{Analysis of the Submodels}

Before analyzing the full model system (5), it is essential to gain insights into the dynamics of the models for HIV only and schistosomiasis only.

3.1. HIV-Only Model. We now consider a model for HIV/AIDS only, obtained by setting $I_{B}=I_{H_{B}}=A_{H_{B}}=$ $A_{H_{T_{B}}}=M=S_{S}=I_{S}=P=0$, so that system in (5) reduces to

$$
\text { HIV/AIDS only }\left\{\begin{array}{l}
\frac{d S_{H}}{d t}=\Lambda_{H}-\left(\lambda_{H}+\mu_{H}\right) S_{H}, \\
\frac{d I_{H}}{d t}=\lambda_{H} S_{H}-\left(\rho+\mu_{H}\right) I_{H}, \\
\frac{d A_{H}}{d t}=\rho I_{H}-\left(\alpha+d_{A}+\mu_{H}\right) A_{H}, \\
\frac{d A_{T_{A}}}{d t}=\alpha A_{H}-\left(\tau d_{A}+\mu_{H}\right) A_{H_{T_{A}}}, \\
\text { with, } \lambda_{H}=\frac{\beta_{H} c\left[I_{H}+\eta A_{H}+\kappa A_{H_{A}}\right]}{N_{H}} \\
N_{H}=S_{H}+I_{H}+A_{H}+A_{H_{T_{A}}} .
\end{array}\right.
$$

For system (7), it can be shown that the region

$$
\Phi_{H}=\left\{\left(S_{H}, I_{H}, A_{H}, A_{H_{T_{A}}}\right) \in \mathbb{R}_{+}^{4}: N_{H} \leq \frac{\Lambda_{H}}{\mu_{H}}\right\}
$$

is invariant and attracting. Thus, the dynamics of the HIVonly model will be considered in $\Phi_{H}$.

3.1.1. Disease-Free Equilibrium and Stability Analysis. Model system (7) has an evident disease-free given by

$$
u_{0 H}=\left(S_{H}^{0}, I_{H}^{0}, A_{H}^{0}, A_{H_{T_{A}}}^{0}\right)=\left(\frac{\Lambda_{H}}{\mu_{H}}, 0,0,0\right) .
$$

Following the next generation approach and the notation defined therein [43], matrices $F$ and $V$ for new infection terms and the remaining transfer terms are, respectively, given by

$$
\begin{gathered}
F=\left[\begin{array}{ccc}
\beta_{H} c & \beta_{H} c \eta & \beta_{H} c \kappa \\
0 & 0 & 0 \\
0 & 0 & 0
\end{array}\right], \\
V=\left[\begin{array}{ccc}
\mu_{\mathrm{H}} & 0 & 0 \\
0 & \mu_{H}+\rho & 0 \\
0 & 0 & \mu_{H}+\tau d_{A}
\end{array}\right] .
\end{gathered}
$$


TABLE 1: Model parameters and their interpretations.

\begin{tabular}{|c|c|c|c|}
\hline Parameter & Symbol & Value & Source \\
\hline Recruitment rate for humans & $\Lambda_{H}$ & $100,000 \mathrm{yr}^{-1}$ & {$[40]$} \\
\hline Natural mortality rate for humans & $\mu_{H}$ & $0.02 \mathrm{yr}^{-1}$ & {$[39,40]$} \\
\hline Natural rate of progression to AIDS & $\rho$ & $0.125 \mathrm{yr}^{-1}$ & {$[40]$} \\
\hline AIDS-related death rate & $d_{A}$ & $0.333 \mathrm{yr}^{-1}$ & [39] \\
\hline Schistosomiasis-related death rate & $d_{B}$ & $0.00201 \mathrm{yr}^{-1}$ & Assume \\
\hline \multicolumn{4}{|l|}{ Product of effective contact rate } \\
\hline \multicolumn{4}{|l|}{ for HIV infection and probability } \\
\hline of HIV transmission per contact & $\beta_{H} c$ & $0.011-0.95 \mathrm{yr}^{-1}$ & {$[39,40]$} \\
\hline \multicolumn{4}{|l|}{ Enhancement factor of schistosomiasis } \\
\hline to HIV infection & $\delta$ & $1.001 \mathrm{yr}^{-1}$ & [39] \\
\hline Modification parameter & $\sigma$ & $1.001 \mathrm{yr}^{-1}$ & {$[39]$} \\
\hline Treatment rate & $\alpha$ & $0.33 \mathrm{yr}^{-1}$ & Assume \\
\hline Recruitment rate for snails & $\Lambda_{S}$ & $10 \mathrm{yr}^{-1}$ & [39] \\
\hline Natural mortality rate from snails & $\mu_{S}$ & $0.072 \mathrm{yr}^{-1}$ & Assume \\
\hline Saturation constant for cercariae & $P_{0}$ & $10^{7}$ & [39] \\
\hline Saturation constant for miracidia & $M_{0}$ & $10^{8}$ & [39] \\
\hline Limitation of the growth velocity & $\epsilon$ & 100 & [39] \\
\hline Number of eggs excreted by humans & $N_{E}$ & 500 & [39] \\
\hline Mortality rate for cercariae & $\mu_{P}$ & $0.504 \mathrm{yr}^{-1}$ & {$[39]$} \\
\hline Mortality rate for miracidia & $\mu_{M}$ & $0.65 \mathrm{yr}^{-1}$ & Assume \\
\hline Snail disease induced death rate & $d_{S}$ & $0.08 \mathrm{yr}^{-1}$ & Assume \\
\hline \multicolumn{4}{|l|}{ Rate at which eggs successfully } \\
\hline become miracidia & $\gamma$ & $0.835 \mathrm{yr}^{-1}$ & [39] \\
\hline \multicolumn{4}{|l|}{ Rate at which sporocysts successfully } \\
\hline become cercariae & $\theta$ & $0.9 \mathrm{yr}^{-1}$ & [39] \\
\hline Modification parameter & $\kappa$ & 0.4 & {$[41]$} \\
\hline Modification parameter & $\eta$ & 1.25 & Assume \\
\hline Modification parameter & $\tau$ & 0.001 & Assume \\
\hline Rate of recovery from schistosomiasis & $\omega, \omega_{1}$ & 0.56 & Assume \\
\hline
\end{tabular}

It follows from (10) that the reproduction number of the system (7) is given by

$$
\mathcal{R}_{A}=\frac{\beta_{H} c\left[\rho \kappa \alpha+\left(\mu_{H}+\tau d_{A}\right)\left(\eta \rho+\alpha+\mu_{H}+d_{A}\right)\right]}{\left(\mu_{H}+\rho\right)\left(\mu_{H}+d_{A}\right)\left(\mu_{H}+\alpha+d_{A}\right)} .
$$

The threshold quantity $\mathcal{R}_{A}$ measures the average number of new secondary cases generated by a single individual in a population where the aforementioned HIV control measures are in place. An associated epidemiological threshold which is the basic reproductive number $\mathcal{R}_{0}$, obtained using the same technique of the next generation operator [43], by considering model system (7) in the absence of HIV intervention strategies, is given by

$$
\mathcal{R}_{0_{A}}=\frac{\beta_{H} c\left(\mu_{H}+d_{A}+\eta \rho\right)}{\left(\mu_{H}+\rho\right)\left(\mu_{H}+d_{A}\right)} .
$$

This disease threshold quantity $\mathcal{R}_{0_{A}}$ measures the average number of new infections generated by a single infected individual in a completely susceptible population where there are no HIV intervention strategies. Using Theorem 2 in [43], the following result is established.

Lemma 3. The disease-free equilibrium $u_{0 H}$ of system (7) is locally asymptotically stable (LAS) if $\mathcal{R}_{A}<1$ and unstable if $\mathcal{R}_{A}>1$.

3.1.2. Sensitivity Analysis of HIV-Only-Induced Reproductive Number. To avoid repetition we refer the reader to a detailed analysis of the reproductive number for model system (7), in the work of Bhunu et al. [44].

3.1.3. Global Stability of HIV/AIDS Model. We claim the following result.

Lemma 4. The disease-free equilibrium $\left(U_{0 H}\right)$ of model system (7) is globally asymptotically stable (GAS) if $\mathcal{R}_{A}<1$ and unstable if $\mathcal{R}_{A}>1$. 
Proof. The proof is based on using a comparison theorem [45]. Note that the equations of the infected components in system (7) can be written as

$$
\begin{aligned}
{\left[\begin{array}{c}
\frac{d I_{H}}{d t} \\
\frac{d A_{H}}{d t} \\
\frac{d A_{H_{T_{A}}}}{d t}
\end{array}\right]=[F-V]\left[\begin{array}{c}
I_{H} \\
A_{H} \\
A_{H_{T_{A}}}
\end{array}\right] } \\
-\beta_{H} c\left[1-\frac{S_{H}}{N_{H}}\right]\left[\begin{array}{lll}
1 & \eta & \kappa \\
0 & 0 & 0 \\
0 & 0 & 0
\end{array}\right]\left[\begin{array}{c}
I_{H} \\
A_{H} \\
A_{H_{T_{A}}}
\end{array}\right],
\end{aligned}
$$

where $F$ and $V$, are as defined earlier in (10). Since $S_{H} \leq N_{H}$, (for all $t \geq 0$ ) in $\Phi_{H}$, it follows that

$$
\left[\begin{array}{c}
\frac{d I_{H}}{d t} \\
\frac{d A_{H}}{d t} \\
\frac{d A_{H_{T_{A}}}}{d t}
\end{array}\right] \leq[F-V]\left[\begin{array}{c}
I_{H} \\
A_{H} \\
A_{H_{T_{A}}}
\end{array}\right] .
$$

Using the fact that the eigenvalues of the matrix $F-V$ all have negative real parts, it follows that the linearized differential inequality system (14) is stable whenever $\mathcal{R}_{A}<1$. Consequently, $\left(I_{H}, A_{H}, A_{H_{T_{A}}}\right) \rightarrow(0,0,0)$ as $t \rightarrow \infty$. Thus, by a comparison theorem $[45]\left(I_{H}, A_{H}, A_{H_{T_{A}}}\right) \rightarrow(0,0,0)$ as $t \rightarrow \infty$, and evaluating system (7) at $I_{\mathrm{H}}=A_{H}=A_{H_{T_{A}}}=0$ gives $S_{H} \rightarrow S_{H}{ }^{0}$ for $\mathcal{R}_{A}<1$. Hence, the DFE $\left(U_{0 H}\right)$ is GAS for $\mathcal{R}_{A}<1$.

3.1.4. HIV-Only Equilibrium. Expressed in terms of the equilibrium value of the force of infection $\lambda_{H}^{*}$, this equilibrium is given by

$u_{1}^{*}\left\{\begin{array}{l}S_{H}^{*}=\frac{\Lambda_{H}}{\mu_{H}+\lambda_{H}^{*}}, \\ I_{H}^{*}=\frac{\Lambda_{H} \lambda_{H}^{*}}{\left(\mu_{H}+\lambda_{H}^{*}\right)\left(\mu_{H}+\rho\right)}, \\ A_{H}^{*}=\frac{\rho \lambda_{H}^{*} \Lambda_{H}}{\left(\mu_{H}+\lambda_{H}^{*}\right)\left(\mu_{H}+\rho\right)\left(\mu_{H}+\alpha+d_{A}\right)}, \\ A_{H_{T_{A}}}^{*}=\frac{\alpha \rho \lambda_{H}^{*} \Lambda_{H}}{\left(\mu_{H}+\lambda_{H}^{*}\right)\left(\mu_{H}+d_{A}\right)\left(\mu_{H}+\rho\right)\left(\mu_{H}+\alpha+d_{A}\right)} .\end{array}\right.$

The local bifurcation analysis is based on the centre manifold approach [46] as described by Theorem 4.1 in [47], stated in the appendix for convenience (also see [43] for more details). To apply the said Theorem 10 in order to establish the local asymptotic stability of the endemic equilibrium, it is convenient to make the following change of variables: $S_{H}=$ $x_{1}, I_{H}=x_{2}, A_{H}=x_{3}$, and $A_{H_{T_{A}}}=x_{4}$, so that $N_{H}=\sum_{n=1}^{4} x_{n}$.
We now use the vector notation $X=\left(x_{1}, x_{2}, x_{3}, x_{4}\right)^{T}$. Then, model system (7) can be written in the form $d X / d t=F=$ $\left(f_{1}, f_{2}, f_{3}, f_{4}\right)^{T}$, where

$$
\begin{aligned}
& x_{1}^{\prime}(t)=f_{1}=\Lambda_{H}-\frac{\beta_{H} c\left(x_{2}+\eta x_{3}+\kappa x_{4}\right)}{\sum_{n=1}^{4} x_{n}} x_{1}-\mu_{H} x_{1}, \\
& x_{2}^{\prime}(t)=f_{2}=\frac{\beta_{H} c\left(x_{2}+\eta x_{3}+\kappa x_{4}\right)}{\sum_{n=1}^{4} x_{n}} x_{1}-\left(\mu_{H}+\rho\right) x_{2}, \\
& x_{3}^{\prime}(t)=f_{3}=\rho x_{2}-\left(\mu_{H}+\alpha+d_{A}\right) x_{3}, \\
& x_{4}^{\prime}(t)=f_{4}=\alpha x_{3}-\left(\mu_{H}+\tau d_{A}\right) x_{4} .
\end{aligned}
$$

The Jacobian matrix of system (16) at $U_{0}$ is given by

$$
\begin{aligned}
& J\left(u_{0 H}\right) \\
& =\left[\begin{array}{cccc}
-\mu_{H} & -\beta_{H} c & -\eta \beta_{H} c & -\kappa \beta_{H} c \\
0 & \beta_{H} c-\left(\mu_{H}+\rho\right) & \eta \beta_{H} c & \kappa \beta_{H} c \\
0 & \rho & -\left(\mu_{H}+\alpha+d_{A}\right) & 0 \\
0 & 0 & \alpha & -\left(\mu_{H}+\tau d_{A}\right)
\end{array}\right],
\end{aligned}
$$

from which it can be shown that the HIV/AIDS-induced reproduction number is

$$
\mathcal{R}_{A}=\frac{\beta_{H} c\left[\kappa \rho \alpha+\left(\mu_{H}+\tau d_{A}\right)\left(\eta \rho+\alpha+\mu_{H}+d_{A}\right)\right]}{\left(\mu_{H}+\rho\right)\left(\mu_{H}+d_{A}\right)\left(\mu_{H}+\alpha+d_{A}\right)} .
$$

If $\beta_{H}$ is taken as a bifurcation parameter and by solving for $\beta_{H}$ when $\mathcal{R}_{A}=1$, we obtain

$$
\beta_{H}=\beta_{H}^{*}=\frac{\left(\mu_{H}+\rho\right)\left(\mu_{H}+d_{A}\right)\left(\mu_{H}+\alpha+d_{A}\right)}{c\left[\kappa \rho \alpha+\left(\mu_{H}+\tau d_{A}\right)\left(\eta \rho+\alpha+\mu_{H}+d_{A}\right)\right]} .
$$

Note that the linearized system of the transformed model (16) with $\beta_{H}=\beta_{H}^{*}$ has a simple zero eigenvalue, which allows the use of Castillo-Chavez and Song result [47] to analyze the dynamics of (16) near $\beta_{H}=\beta_{H}^{*}$. It can be shown that the Jacobian of (16) at $\beta_{H}=\beta_{H}^{*}$ has a right eigenvector associated with the zero eigenvalue given by $u=$ $\left[u_{1}, u_{2}, u_{3}, u_{4}\right]^{T}$, where

$$
\begin{aligned}
& u_{1}=\frac{-\beta_{H} c\left(u_{2}+\eta u_{3}+\kappa u_{4}\right)}{\mu_{H}}, \quad u_{2}>0, \\
& u_{3}=\frac{\rho u_{2}}{\alpha+d_{A}+\mu_{H}}, \quad u_{4}=\frac{\alpha u_{3}}{\mu_{H}+\tau d_{A}} .
\end{aligned}
$$

The left eigenvector of $J\left(U_{0 H}\right)$ associated with the zero eigenvalue at $\beta_{H}=\beta_{H}^{*}$ is given by $v=\left[v_{1}, v_{2}, v_{3}, v_{4}\right]^{T}$, where

$$
v_{1}=0, \quad v_{2}=\frac{\rho v_{3}}{\mu_{H}+\rho-\beta_{H}^{*} c}, \quad v_{3}>0, \quad v_{4}=\frac{\kappa \beta_{H} c v_{2}}{\mu_{H}+\tau d_{A}} .
$$


Computation of the Bifurcation Parameters $a$ and $b$. The application of Theorem 10 (see the appendix) entails the computation of two parameters $a$ and $b$, say. After some little algebraic manipulations and rearrangements, it can be shown that

$$
a=-\frac{2 \beta_{H}^{*} c \mu_{H} v_{2}}{\Lambda_{H}}\left(u_{2}+u_{3}+u_{4}\right)\left(u_{2}+\eta u_{3}+\kappa u_{4}\right)<0 .
$$

Furthermore,

$$
b=c\left(u_{2}+\eta u_{3}+\kappa u_{4}\right) v_{2}>0 .
$$

This sign of $b$ may be expected in general for epidemic models because, in essence, using $\beta$ as a bifurcation parameter often ensures $b>0$ [43]. Since $a<0$ (which excludes any possibility of multiple equilibria and hence backward bifurcation), model system (16) has a forward (or transcritical) bifurcation at $\mathcal{R}_{A}=1$, and consequently, the local stability implies global stability. This result is summarized below.

Theorem 2. The endemic equilibrium $\boldsymbol{U}_{1}^{*}$ is locally asymptotically stable for $\mathcal{R}_{A}>1$.

3.2. Schistosomiasis-Only Model. In the absence of HIV/ AIDS in the community (obtained by setting HIV/AIDSrelated parameters to zero from system (5)) schistosomiasisonly model is given by

Schistosomiasis-only model $\left\{\begin{array}{l}\frac{d S_{H}}{d t}=\Lambda_{H}+\omega I_{B}-\left(\lambda_{P}+\mu_{H}\right) S_{H}, \\ \frac{d I_{B}}{d t}=\lambda_{H} I_{B}-\left(\mu_{H}+\omega+d_{B}\right) I_{B}, \\ \frac{d M}{d t}=N_{E} \gamma I_{B}-\mu_{M} M, \\ \frac{d S_{S}}{d t}=\Lambda_{S}-\lambda_{M} S_{S}-\mu_{S} S_{S}, \\ \frac{d I_{S}}{d t}=\lambda_{M} S_{S}-\left(\mu_{S}+d_{S}\right) I_{S}, \\ \frac{d P}{d t}=\theta I_{S}-\mu_{P} P, \\ \text { with, } \lambda_{P}=\frac{\beta_{P} P(t)}{P_{0}+\epsilon P(t)}, \\ \quad \lambda_{M}=\frac{\beta_{M} M(t)}{M_{0}+\epsilon M(t)} .\end{array}\right.$

For system (24), it can be shown that the region

$$
\Phi_{B}=\left\{\begin{array}{l}
\left(S_{H}, I_{B}\right) \in \mathbb{R}_{+}^{2}: N_{H} \leq \frac{\Lambda_{H}}{\mu_{H}}, \\
M \in \mathbb{R}_{+}: M \leq \frac{\gamma \Lambda_{H} N_{E}(1+\sigma)}{\mu_{M} \mu_{H}}, \\
\left(S_{S}, I_{S}\right) \in \mathbb{R}_{+}^{2}: N_{S} \leq \frac{\Lambda_{S}}{\mu_{S}}, \\
P \in \mathbb{R}_{+}: P \leq \frac{\theta \Lambda_{S}}{\mu_{P} \mu_{S}}
\end{array}\right.
$$

is invariant and attracting. Thus, the dynamics of schistosomiasis-only model will be considered in $\Phi_{B}$.

3.2.1. Disease-Free Equilibrium and Stability Analysis. Model system (24) has an evident disease-free given by

$$
U_{0 B}=\left(S_{H}^{0}, I_{B}^{0}, M^{0}, S_{S}^{0}, I_{S}^{0}, P^{0}\right)=\left(\frac{\Lambda_{H}}{\mu_{H}}, 0,0, \frac{\Lambda_{S}}{\mu_{S}}, 0,0\right) .
$$

Following van den Driessche and Watmough [43], the reproduction number of the model system (24) is given by

$$
\begin{aligned}
\mathcal{R}_{B} & =\sqrt{\left(\frac{\beta_{p} N_{E} \gamma \Lambda_{H}}{\mu_{M} \mu_{H} P_{0}\left(\mu_{H}+\omega+d_{B}\right)}\right)\left(\frac{\beta_{M} \theta \Lambda_{S}}{\mu_{P} \mu_{S} M_{0}\left(\mu_{S}+d_{S}\right)}\right)} \\
& =\sqrt{\mathcal{R}_{H} \mathcal{R}_{S}}
\end{aligned}
$$

where $\mathcal{R}_{H}=\beta_{p} N_{E} \gamma \Lambda_{H} / \mu_{M} \mu_{H} P_{0}\left(\mu_{H}+\omega+d_{B}\right)$ represents the snail-man initial disease transmission and $\mathcal{R}_{S}=$ $\beta_{M} \theta \Lambda_{S} / \mu_{P} \mu_{S} M_{0}\left(\mu_{S}+d_{S}\right)$ is the man-snail initial disease transmission.

The threshold quantity $\mathcal{R}_{B}$ measures the average number of new secondary cases generated by a single individual in a population where there is schistosomiasis treatment. An associated epidemiological threshold, $\mathcal{R}_{0_{B}}$, obtained using a similar technique of the next generation by considering model system (24) in the absence of schistosomiasis treatment is given by

$$
\begin{aligned}
\mathcal{R}_{0_{B}} & =\sqrt{\left(\frac{\beta_{p} N_{E} \gamma \Lambda_{H}}{\mu_{M} \mu_{H} P_{0}\left(\mu_{H}+d_{B}\right)}\right)\left(\frac{\beta_{M} \theta \Lambda_{S}}{\mu_{P} \mu_{S} M_{0}\left(\mu_{S}+d_{S}\right)}\right)} \\
& =\sqrt{\mathcal{R}_{0_{H}} \mathcal{R}_{0_{S}}}
\end{aligned}
$$

where $\mathcal{R}_{0_{H}}=\beta_{p} N_{E} \gamma \Lambda_{H} / \mu_{M} \mu_{H} P_{0}\left(\mu_{H}+d_{B}\right)$ represents the snail-man initial disease transmission and $\mathcal{R}_{0_{S}}=\beta_{M} \theta \Lambda_{S}$ / $\mu_{P} \mu_{S} M_{0}\left(\mu_{S}+d_{S}\right)$ is the man-snail initial disease transmission. Using Theorem 2 in [43], the following result is established.

Theorem 3. The disease-free equilibrium $\mathcal{U}_{0_{B}}$ is locally asymptotically stable whenever $\mathcal{R}_{B}<1$ and unstable otherwise.

Impact of Schistosomiasis Treatment in the Community. Here, the reproductive number $\mathcal{R}_{B}$ is analyzed to determine whether or not treatment of schistosomiasis patients (modeled by the rate $\omega$ ) can lead to the effective control of schistosomiasis in the community. It follows from (27) that the elasticity [48] of $\mathcal{R}_{B}$ with respect to $\omega$ can be computed using the approach in [49] as follows:

$$
\frac{\omega}{\mathcal{R}_{B}} \frac{\partial \mathcal{R}_{B}}{\partial \omega}=-\frac{\omega}{2\left(\mu_{H}+\omega+d_{B}\right)}<0
$$


The sensitivity index of the reproduction number is used to assess the impact on the relevant parameters to disease transmission. That is, the elasticity measures the effect a change in $\omega$, say, has as a proportional change in $\mathcal{R}_{B}$, and from (29), we note that an increase in $\omega$ will lead to a decrease in $\mathcal{R}_{B}$, thus (29) suggests that an increase in treatment of schistosomiasis patients does have a positive impact in controlling schistosomiasis in the community (assuming full compliance to the therapy, no treatment failure, and no development of resistance).

3.3. Global Stability of the Disease-Free Equilibrium. We shall use the following theorem of Castillo-Chavez et al. [50] in the sequel.

Theorem 4 (see [50]). If system (5) can be written in the form

$$
\begin{gathered}
\frac{d X}{d t}=F(\mathbf{x}, Z), \\
\frac{d Z}{d t}=G(X, Z), \quad G(\mathbf{x}, 0)=0,
\end{gathered}
$$

where $X \in \mathbb{R}^{m}$ denotes (its components) the number of uninfected individuals, $Z \in \mathbb{R}^{n}$ denotes (its components) the number of infected individuals including latent and infectious, and $\mathbf{U}_{0}=\left(\mathbf{x}^{*}, 0\right)$ denotes the disease-free equilibrium of the system. Assume that (i) for $d X / d t=F(X, 0), X^{*}$ is globally asymptotically stable, (ii) $G(X, Z)=A Z-\hat{G}(X, Z)$, $\hat{G}(X, Z) \geq 0$ for $(X, Z) \in \mathcal{D}$, where $A=D_{Z} G\left(X^{*}, 0\right)$ is an $M$-matrix (the off-diagonal elements of $A$ are nonnegative) and $\mathcal{D}$ is the region where the model makes biological sense. Then the fixed point $\mathbf{U}_{0}=\left(\mathbf{x}^{*}, 0\right)$ is a globally asymptotic stable equilibrium of model system (5) provided $\mathcal{R}_{B}<1$.

Applying Theorem 4 to model system (5) yields

$$
\widehat{G}(X, Y)=\left[\begin{array}{c}
\widehat{G_{1}}(X, Y) \\
\widehat{G_{2}}(X, Y) \\
\widehat{G_{3}}(X, Y) \\
\widehat{G_{4}}(X, Y)
\end{array}\right]=\left[\begin{array}{c}
\beta_{P} P\left(\frac{\Lambda_{H}}{P_{0} \mu_{H}}-\frac{S_{H}}{P_{0}+\epsilon P}\right) \\
\beta_{M} M\left(\frac{\Lambda_{S}}{M_{0} \mu_{S}}-\frac{S_{S}}{M_{0}+\epsilon M}\right) \\
0 \\
0
\end{array}\right] .
$$

Since $S_{H}^{0}\left(=\Lambda_{H} / \mu_{H}\right)\left(1 / P_{0}\right) \geq S_{H} /\left(P_{0}+\epsilon P\right)$ and $S_{S}\left(=\Lambda_{S} /\right.$ $\left.\mu_{S}\right)\left(1 / M_{0}\right) \geq S_{S} /\left(M_{0}+\epsilon M\right)$, it follows that $\hat{G}(X, Y) \geq 0$. We summarise the result in Theorem 5 .

Theorem 5. The disease-free equilibrium $\left(U_{0 B}\right)$ of model system (24) is globally asymptotically stable (GAS) if $\mathcal{R}_{B}<1$ and unstable if $\mathcal{R}_{B}>1$.
3.3.1. Schistosomiasis-Only Equilibrium. Model system (24) has an endemic equilibrium denoted by $\mathcal{U}_{2}^{*}$, where

$$
\left\{\begin{array}{l}
S_{H}^{* *}=\frac{\Lambda_{H}}{\mu_{H}+\omega+\lambda_{P}^{* *}}, \\
I_{B}^{* *}=\frac{\Lambda_{H} \lambda_{P}^{* *}}{\left(\mu_{H}+\omega+\lambda_{P}^{* *}\right)\left(\mu_{H}+\omega+d_{B}\right)}, \\
M^{* *}=\frac{N_{E} \gamma \Lambda_{H} \lambda_{P}^{* *}}{\mu_{M}\left(\mu_{H}+\omega+\lambda_{P}^{* *}\right)\left(\mu_{H}+\omega+d_{B}\right)}, \\
S_{S}^{* *}=\frac{\Lambda_{S}}{\mu_{S}+\lambda_{S}^{* *}}, \\
I_{S}^{* *}=\frac{\Lambda_{S} \lambda_{M}^{* *}}{\left(\mu_{S}+\lambda_{M}^{* *}\right)\left(\mu_{S}+d_{S}\right)}, \\
P^{* *}=\frac{\theta \Lambda_{S} \lambda_{S}^{* *}}{\mu_{P}\left(\mu_{S}+\lambda_{S}^{* *}\right)\left(\mu_{S}+d_{S}\right)}, \\
\text { with } \lambda_{P}^{* *}=\frac{\beta_{P} P^{* *}}{P_{0}+\epsilon P^{* *}}, \quad \lambda_{M}^{* *}=\frac{\beta_{M} M^{* *}}{M_{0}+\epsilon M^{* *}}
\end{array} .\right.
$$

The local asymptotic stability of the endemic equilibrium $u_{2}^{*}$ can also be analyzed using the centre manifold theory. In this case, the Jacobian matrix of the system at $U_{0 B}$ is given by

$J\left(u_{0 B}\right)$
$\left[\begin{array}{cccccc}-\mu_{H} & 0 & 0 & 0 & 0 & -\frac{\beta_{P} \Lambda_{H}}{P_{0} \mu_{H}} \\ 0 & -\left(\mu_{H}+\omega+d_{B}\right) & 0 & 0 & 0 & \frac{\beta_{P} \Lambda_{H}}{P_{0} \mu_{H}} \\ 0 & N_{E} \gamma & -\mu_{M} & 0 & 0 & 0 \\ 0 & 0 & -\frac{\beta_{M} \Lambda_{S}}{M_{0} \mu_{S}} & -\mu_{S} & 0 & 0 \\ 0 & \frac{\beta_{M} \Lambda_{S}}{M_{0} \mu_{S}} & 0 & -\left(\mu_{S}+d_{S}\right) & 0 \\ 0 & 0 & 0 & \theta & -\mu_{P}\end{array}\right]$.

If $\beta_{P}$ is taken as a bifurcation parameter, and solving for $\beta_{P}$ when $\mathcal{R}_{B}=1$, we obtain

$$
\beta_{P}=\beta_{P}^{*}=\frac{\mu_{M} \mu_{H} P_{0}\left(\mu_{H}+\omega+d_{B}\right)}{N_{E} \gamma \Lambda_{H} \mathcal{R}_{S}} .
$$

The linearized system of the the model with $\beta_{P}=\beta_{P}^{*}$ has a simple zero eigenvalue. Therefore, it can be shown that the 
above Jacobian has a right eigenvector given by $w=\left[w_{1}, w_{2}\right.$, $\left.w_{3}, w_{4}, w_{5}, w_{6}\right]^{T}$, where

$$
\begin{gathered}
w_{1}=-\frac{\beta_{P}^{*} \Lambda_{H} \mathcal{R}_{0_{S}} w_{3}}{P_{0} \mu_{H}^{2}}, \quad w_{2}=\frac{\mu_{P} \beta_{P}^{*} \Lambda_{H} w_{3}}{\theta\left(\mu_{H}+\omega+d_{B}\right)}, \quad w_{3}=w_{3}, \\
w_{4}=-\frac{\beta_{M} \Lambda_{S} w_{3}}{M_{0} \mu_{S}^{2}}, \quad w_{5}=\frac{\beta_{M} \Lambda_{S} w_{3}}{\left(\mu_{S}+d_{S}\right) M_{0} \mu_{S}}, \quad w_{6}=\mathcal{R}_{0_{S}} w_{3} .
\end{gathered}
$$

The left eigenvector of $J\left(U_{0_{B}}\right)$ associated with the zero eigenvalue at $\beta_{P}=\beta_{P}^{*}$ is given by $z=\left[z_{1}, z_{2}, z_{3}, z_{4}, z_{5}, z_{6}\right]^{T}$, where

$$
\begin{gathered}
z_{1}=0=z_{4}, \quad z_{3}>0, \quad z_{2}=\frac{N_{E} \gamma z_{3}}{\mu_{H}+\omega+d_{B}}, \\
z_{5}=\frac{\mu_{M} M_{0} \mu_{S} z_{3}}{\beta_{M} \Lambda_{S}}, \quad z_{6}=\frac{\beta_{P}^{*} \Lambda_{H} N_{E} \gamma z_{3}}{P_{0} \mu_{H} \mu_{P}\left(\mu_{H}+\omega+d_{B}\right)} .
\end{gathered}
$$

Computation of the bifurcation coefficients $a$ and $b$ yields

$$
\begin{aligned}
& a=-2 z_{3} w_{3}^{2}( \frac{N_{E} \gamma \mathcal{R}_{S}^{2} \beta_{P}^{*} \Lambda_{H}\left(\beta_{P}^{*}+\epsilon \mu_{H}\right)}{\left(\mu_{H}+\omega+d_{B}\right) P_{0}^{2} \mu_{H}^{2}} \\
&\left.+\frac{N_{E} \gamma \theta \beta_{P}^{*} \Lambda_{H} \beta_{M} \Lambda_{S}\left(\beta_{M}+\epsilon \mu_{S}\right)}{\left(\mu_{H}+\omega+d_{B}\right)\left(\mu_{S}+d_{S}\right) P_{0} \mu_{H} M_{0}^{2} \mu_{S}^{2}}\right)<0, \\
& b=\frac{N_{E} \gamma \mathcal{R}_{S} \Lambda_{S} z_{3} w_{3}}{\left(\mu_{H}+\omega+d_{B}\right) P_{0} \mu_{S}}>0 .
\end{aligned}
$$

Thus, the following result is established.

Theorem 6. The unique endemic equilibrium $\mathcal{U}_{2}^{*}$ is locally asymptotically stable for $\mathcal{R}_{B}>1$. ity.

Since $a<0$, local stability of $u_{2}^{*}$ implies its global stabil-

\section{HIV/AIDS and Schistosomiasis Model}

Model system (5) has evident disease-free (DFE) given by

$$
\begin{aligned}
\mathcal{U}_{0} & =\left(S_{H}^{0}, I_{B}^{0}, I_{H}^{0}, A_{H}^{0}, A_{H_{T_{A}}}^{0}, I_{H_{B}}^{0}, A_{H_{B}}^{0}, A_{H_{T_{B}}}^{0}, M^{0}, S_{S}^{0}, I_{S}^{0}, P^{0}\right) \\
& =\left(\frac{\Lambda_{H}}{\mu_{H}}, 0,0,0,0,0,0,0,0, \frac{\Lambda_{S}}{\mu_{S}}, 0,0\right) .
\end{aligned}
$$

Following van den Driessche and Watmough [43], the reproduction number of the model is

$$
\mathcal{R}_{H_{B}}=\max \left\{\mathcal{R}_{A}, \mathcal{R}_{B}\right\}
$$

with $\mathcal{R}_{A}$ and $\mathcal{R}_{B}$ defined as earlier in Section 3 above. Using Theorem 2 in [43], the following result is established.

Theorem 7. The disease-free equilibrium $\mathcal{U}_{0}$ is locally asymptotically stable whenever $\mathcal{R}_{H_{B}}<1$ and unstable otherwise.

4.1. Sensitivity Analysis. In this section we investigate the effects of HIV/AIDS on schistosomiasis and vice versa, in the presence and absence of the aforementioned intervention strategies.

Impact of Schistosomiasis on HIV/AIDS in the Absence of Control Measures. To analyze the effects of schistosomiasis on HIV/AIDS and vice versa in the absence of control measures for either HIV/AIDS or schistosomiasis, we begin by introducing the following notation; in the absence of antiretroviral therapy $(\alpha=0)$ the reproductive number is denoted by $\mathcal{R}_{0_{A}}$ and also in the absence of schistosomiasis treatment $(\omega=0), \mathcal{R}_{B}=\mathcal{R}_{0_{B}}$. Thus, to express $\mathcal{R}_{0_{B}}$ in terms of $\mathcal{R}_{0_{A}}$, we solve for $\mu_{H}$ and obtain

$$
\mu_{H}=\frac{-\left(\phi_{1} \mathcal{R}_{0_{A}}+\phi_{2}\right)+\sqrt{\phi_{3} \mathcal{R}_{0_{A}}^{2}+\phi_{4} \mathcal{R}_{0_{A}}+\phi_{5}}}{2 \mathcal{R}_{0_{A}}},
$$

where

$$
\begin{aligned}
& \phi_{1}=\rho+d_{A}, \\
& \phi_{2}=-\beta_{H} c, \\
& \phi_{3}=\left(\rho-d_{A}\right)^{2}, \\
& \phi_{4}=2 \beta_{H} c\left(d_{A}+\rho(2 \eta-1)\right), \\
& \phi_{5}=\left(\beta_{H} c\right)^{2} .
\end{aligned}
$$

Let $\sqrt{\phi_{3} \mathcal{R}_{0_{A}}^{2}+\phi_{4} \mathcal{R}_{0_{A}}+\phi_{5}}=\phi_{6} \mathcal{R}_{0_{A}}+\phi_{7}$, then, (40) becomes

$$
\mu_{H}=\frac{\left(\phi_{6}-\phi_{1}\right) \mathcal{R}_{0_{A}}+\left(\phi_{7}-\phi_{2}\right)}{2 \mathcal{R}_{0_{A}}}
$$

Substituting (42) into the expression for $\mathcal{R}_{0_{B}}$, we have

$$
\mathcal{R}_{0_{B}}^{2}=\frac{4 \mathcal{R}_{0_{S}} \mathcal{R}_{0_{A}}^{2} \beta_{P} N_{E} \gamma \Lambda_{H}}{\mu_{M} P_{0}\left[\left(\left(\phi_{6}-\phi_{1}\right) \mathcal{R}_{0_{A}}+\left(\phi_{7}-\phi_{2}\right)\right)^{2}+2 d_{A} \mathcal{R}_{0_{A}}\left(\left(\phi_{6}-\phi_{1}\right) \mathcal{R}_{0_{A}}+\left(\phi_{7}-\phi_{2}\right)\right)\right]} .
$$

Differentiating $\mathcal{R}_{0_{B}}$ partially with respect to $\mathcal{R}_{0_{A}}$ yields

$$
\frac{\partial \mathcal{R}_{0_{B}}}{\partial \mathcal{R}_{0_{A}}}=\frac{4 \mathcal{R}_{0_{S}} \mathcal{R}_{0_{A}} \beta_{P} N_{E} \gamma \Lambda_{H}\left(\mathcal{R}_{0_{A}}\left(\phi_{7}-\phi_{2}\right)\left(\phi_{6}-\phi_{1}-d_{A}\right)+\left(\phi_{7}-\phi_{2}\right)^{2}\right)}{\mu_{M} P_{0} \mathcal{R}_{0_{B}}\left[\left(\left(\phi_{6}-\phi_{1}\right) \mathcal{R}_{0_{A}}+\left(\phi_{7}-\phi_{2}\right)\right)^{2}+2 d_{A} \mathcal{R}_{0_{A}}\left(\left(\phi_{6}-\phi_{1}\right) \mathcal{R}_{0_{A}}+\left(\phi_{7}-\phi_{2}\right)\right)\right]^{2}} .
$$


Now, whenever (44) is greater than zero, an increase in HIV/AIDS cases results in an increase of schistosomiasis cases in the community. If (44) is equal to zero, this implies that HIV/AIDS cases have no effect on the transmission dynamics of schistosomiasis. Setting $\mathcal{R}_{0_{B}}=1$ and expressing $\mu_{H}$ as the subject of formula, we have

$$
\mu_{H}=\frac{-d_{B} \theta_{1} \mathcal{R}_{0_{B}}+\sqrt{\left(\theta_{1} d_{B} \mathcal{R}_{0_{B}}\right)^{2}+4 \theta_{2}}}{2 \theta_{1} \mathcal{R}_{0_{B}}},
$$

where $\theta_{1}=\mu_{M} P_{0}$ and $\theta_{2}=\mu_{M} P_{0} \beta_{P} N_{E} \gamma \Lambda_{H} \mathcal{R}_{0_{S}}$. Consider $\sqrt{\left(\theta_{1} d_{B} \mathcal{R}_{0_{B}}\right)^{2}+4 \theta_{2}}=\theta_{3} \mathcal{R}_{0_{B}}+\theta_{4}$ such that $\left(\theta_{3}-d_{B} \theta_{1}\right) \mathcal{R}_{0_{B}}+$ $\theta_{4}>0$. Then, $\mathcal{R}_{0_{A}}$ expressed in terms of $\mathcal{R}_{0_{B}}$ reads

$$
\mathcal{R}_{0_{A}}=\frac{2 \theta_{1} \beta_{H} c\left(\kappa \mathcal{R}_{0_{B}}^{2}+\theta_{4} \mathcal{R}_{0_{B}}\right)}{h_{1} \mathcal{R}_{0_{B}}^{2}+h_{2} \mathcal{R}_{0_{B}}+h_{3}}
$$

where

$$
\begin{aligned}
& h_{1}=\left(\theta_{3}-d_{B} \theta_{1}\right)^{2}+4 \rho d_{A} \theta_{1}^{2}+2 \theta_{1}\left(\theta_{3}-d_{B} \theta_{1}\right)\left(\rho+d_{A}\right)>0, \\
& h_{2}=2 \theta_{4}\left(\theta_{3}-d_{B} \theta_{1}\right)+2 \theta_{1} \theta_{4}\left(\rho+d_{A}\right)>0, \\
& h_{3}=\theta_{4}^{2}>0, \\
& \kappa_{1}=\theta_{3}+\theta_{1}\left(2 \eta \rho+2 d_{A}-d_{B}\right)>0 .
\end{aligned}
$$

Partially differentiating $\mathcal{R}_{0_{A}}$ with respect to $\mathcal{R}_{0_{B}}$ yields

$$
\frac{\partial \mathcal{R}_{0_{A}}}{\partial \mathcal{R}_{0_{B}}}=\frac{\left(\kappa_{1} h_{2}-\theta_{4} h_{1}\right) \mathcal{R}_{0_{B}}^{2}+2 \kappa_{1} h_{3} \mathcal{R}_{0_{B}}+\theta_{4} h_{3}}{\left(h_{1} \mathcal{R}_{0_{B}}^{2}+h_{2} \mathcal{R}_{0_{B}}+h_{3}\right)^{2}} .
$$

Thus, whenever $\kappa_{1} h_{2} \geq \theta_{4} h_{1}$, (48) is strictly positive meaning that schistosomiasis enhances HIV infection as a damaged urethra has increased chances of HIV entering the blood stream. The relationship between the HIV/AIDS basic reproduction number and the schistosomiasis basic reproduction number is illustrated graphically in Figure 2 using parameter values from Table 1.

The graph in Figure 2 shows that an increase in the schistosomiasis-induced basic reproduction number results in an increase of the HIV/AIDS-induced basic reproduction number, suggesting that infection by schistosomiasis enhances the chances of HIV infection per sexual contact. This is as a result of the eggs of the parasites causing injury in the reproductive organs which enhance the transmission of sexually transmitted diseases such as HIV/AIDS and Gonorrhoea [51]. Thus, schistosomiasis control has a positive impact in controlling the transmission dynamics of HIV/AIDS.

Impact of Schistosomiasis Treatment on HIV/AIDS. Expressing $\mathcal{R}_{0 B}$ in terms of $\mathcal{R}_{B}$, we obtain

$$
\mathcal{R}_{0_{B}}=\frac{\left(\mu_{H}+\omega+d_{B}\right) \mathcal{R}_{B}}{\left(\mu_{H}+d_{B}\right)} .
$$

Substituting (49) into (46) yields

$$
\mathcal{R}_{0_{A}}=\frac{2 \beta_{H} c \theta_{1} \mathcal{R}_{B}\left[\left(\mu_{H}+\omega+d_{B}\right)\left(\theta_{4}\left(\mu_{H}+d_{B}\right)+\left(\mu_{H}+\omega+d_{B}\right) \kappa_{1} \mathcal{R}_{B}\right]\right.}{\left(\mu_{H}+\omega+d_{B}\right)^{2} h_{1} \mathcal{R}_{B}^{2}+\left(\mu_{H}+d_{B}\right)\left(\mu_{H}+\omega+d_{B}\right) h_{2} \mathcal{R}_{B}+\left(\mu_{H}+d_{B}\right)^{2} h_{3}} .
$$

Partially differentiating $\mathcal{R}_{0_{A}}$ with respect to $\omega$, we have

$$
\frac{\partial \mathcal{R}_{0_{A}}}{\partial \omega}=-\frac{2 \beta_{H} c \theta_{1} k_{3}}{k_{4}}[\Theta-1],
$$

where $\Theta=k_{1} k_{2} / k_{3} k_{4}$, with

$$
\begin{aligned}
& k_{1}=\mathcal{R}_{B}\left[\left(\mu_{H}+d_{B}\right) h_{2}+2 \zeta h_{1} \mathcal{R}_{B}\right], \\
& k_{2}=\zeta \mathcal{R}_{B}\left[\left(\mu_{H}+d_{B}\right) \theta_{4}+\zeta \kappa_{1} \mathcal{R}_{B}\right], \\
& k_{3}=\mathcal{R}_{B}\left[\left(\mu_{H}+d_{B}\right) \theta_{4}+2 \zeta \kappa_{1} \mathcal{R}_{B}\right], \\
& k_{4}=\zeta \mathcal{R}_{B}\left[\zeta h_{1} \mathcal{R}_{B}+\left(\mu_{H}+d_{B}\right) h_{2}\right]+h_{3}, \\
& \zeta=\left(\mu_{H}+\omega+d_{B}\right) .
\end{aligned}
$$

Since $\mathcal{R}_{0_{A}}$ is a decreasing function of $\omega$, schistosomiasis treatment will have a positive impact on the dynamics of HIV/AIDS if $\Theta>1$, no impact if $\Theta=1$, and a negative impact if $\Theta<1$. We summarize the result in lemma 5 .
Lemma 5. Schistosomiasis (bilharzia) treatment for model system (5) only, will have

(i) a positive impact on schistosomiasis and HIV/AIDS coinfection control if $\Theta>1$,

(ii) no impact on schistosomiasis and HIV/AIDS coinfection control if $\Theta=1$,

(iii) a negative impact on schistosomiasis and HIV/AIDS coinfection control if $\Theta<1$.

The synergy between HIV and other diseases such as schistosomiasis provides more opportunities to combat HIV/AIDS by treating its coinfections with these other diseases.

4.2. Global Stability of the Disease-Free Equilibrium $\left(\mathcal{U}_{0}\right)$. We shall use the following theorem of Castillo-Chavez et al. [50] in the sequel. 
Theorem 8 (see [50]). If system (5) can be written in the form

$$
\begin{gathered}
\frac{d X}{d t}=F(\mathbf{x}, Z), \\
\frac{d Z}{d t}=G(X, Z), \quad G(\mathbf{x}, 0)=0,
\end{gathered}
$$

where $X \in \mathbb{R}^{m}$ denotes (its components) the number of uninfected individuals, $Z \in \mathbb{R}^{n}$ denotes (its components) the number of infected individuals including latent, infectious, and so forth, $\mathbf{U}_{0}=\left(\mathbf{x}^{*}, 0\right)$ denotes the disease-free equilibrium of the system. Assume that (i) for $d X / d t=F(X, 0), X^{*}$ is globally asymptotically stable, (ii) $G(X, Z)=A Z-\widehat{G}(X, Z)$, $\widehat{G}(X, Z) \geq 0$ for $(X, Z) \in \mathscr{D}$, where $A=D_{Z} G\left(X^{*}, 0\right)$ is an $M$ matrix (the off-diagonal elements of $A$ are nonnegative) and $D$ is the region where the model makes biological sense. Then the fixed point $\mathbf{U}_{0}=\left(\mathbf{x}^{*}, 0\right)$ is a globally asymptotic stable equilibrium of model system (5) provided $\mathcal{R}_{H_{B}}<1$.

Applying Theorem 8 to model system (5) yields

$$
\begin{aligned}
& \widehat{G}(X, Y) \\
& =\left[\begin{array}{c}
\widehat{G_{1}}(X, Y) \\
\widehat{G_{2}}(X, Y) \\
\widehat{G_{3}}(X, Y) \\
\widehat{G_{4}}(X, Y) \\
\widehat{G_{5}}(X, Y) \\
\widehat{G_{6}}(X, Y) \\
\widehat{G_{7}}(X, Y) \\
\widehat{G_{8}}(X, Y) \\
\widehat{G_{9}}(X, Y) \\
\widehat{G_{10}}(X, Y)
\end{array}\right]=\left[\begin{array}{c}
\delta \lambda_{H} I_{B}+\beta_{P}\left(\frac{P(t) \Lambda_{H}}{P_{0} \mu_{H}}-\frac{P(t) S_{H}(t)}{P_{0}+\epsilon P(t)}\right) \\
\lambda_{P} I_{H}+N_{H}\left(1-\frac{S_{H}}{N_{H}}\right) \\
\lambda_{P} A_{H} \\
\lambda_{P} A_{H_{T_{A}}} \\
-\lambda_{P} I_{H}-\delta \lambda_{H} I_{B} \\
-\lambda_{P} A_{H} \\
-\lambda_{P} A_{H_{T_{A}}} \\
0 \\
\beta_{M}\left(\frac{M \Lambda_{S}}{M_{0} \mu_{S}}-\frac{M S_{S}}{M_{0}+\epsilon M}\right) \\
0
\end{array}\right] .
\end{aligned}
$$

The fact that $\widehat{G_{4}}(X, Y)<0, \widehat{G_{5}}(X, Y)<0$, and $\widehat{G_{6}}(X, Y)<$ 0 implies that $\hat{G}(X, Y)$ may not be greater or equal to zero. Consequently, $U_{0}$ may not be globally asymptotically stable for $\mathcal{R}_{H_{B}}<1$. This suggests the possible existence of multiple equilibria.

4.3. Endemic Equilibria and Its Stability. For model system (5), there are three possible endemic equilibria: the case where there is HIV only, the case where there is schistosomiasis only (which have been discussed in Section 3), and the case when both schistosomiasis and HIV coexist.

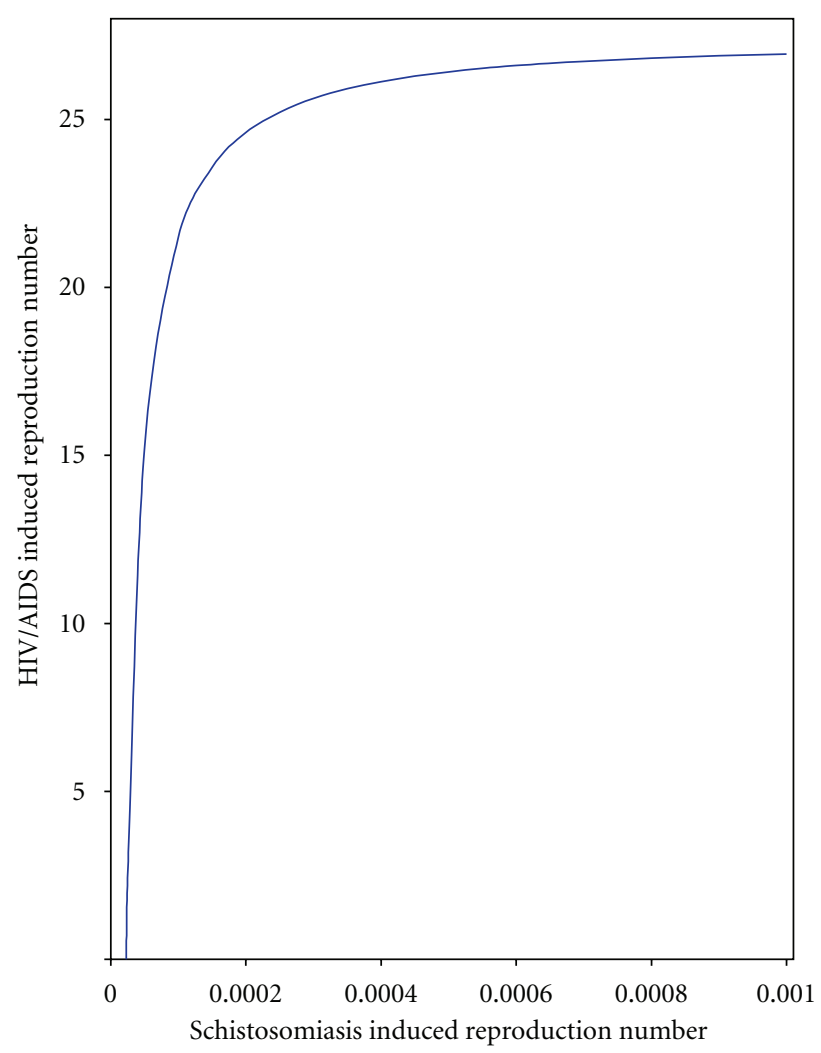

FIGURE 2: Relationship between the HIV/AIDS and the schistosomiasis basic reproduction numbers.

4.3.1. Interior Endemic Equilibrium. This occurs when both infections coexist in the community. The interior equilibrium is given by

$$
\begin{gathered}
u_{3}^{*}=\left(S_{H}^{* * *}, I_{B}^{* * *}, I_{H}^{* * *}, A_{H}^{* * *}, A_{H T_{A}}^{* * *}, I_{H_{B}}^{* * *}, A_{H_{B}}^{* * *},\right. \\
\left.A_{H T_{B}}^{* * *} M^{* * *}, S_{S}^{* * *}, I_{S}^{* * *}, P^{* * *}\right) .
\end{gathered}
$$

The local asymptotic stability of this endemic equilibrium can be analyzed using the centre manifold theory similar to the analysis of $U_{1}^{*}$ and $U_{2}^{*}$, but it is not done here to avoid repetition. Thus, we claim the following result for the stability of $u_{1}^{*}$ and $u_{2}^{*}$.

Theorem 9. If $\mathcal{R}_{H_{B}}>1$ with $\mathcal{R}_{B}>1$ and $\mathcal{R}_{A}>1$, then, the endemic equilibrium point $U_{3}$ is locally asymptotically stable whenever $\mathcal{R}_{H_{B}}>1$.

\section{Numerical Simulations}

In order to illustrate the results of the foregoing analysis, numerical simulations of the full HIV-schistosomiasis model are carried out, using parameter values given in Table 1. The scarcity of data on HIV schistosomiasis codynamics limits our ability to calibrate, but, for the purpose of illustration, other parameter values are assumed. These parsimonious assumptions reflect the lack of information currently available on the coinfection of the two diseases. 


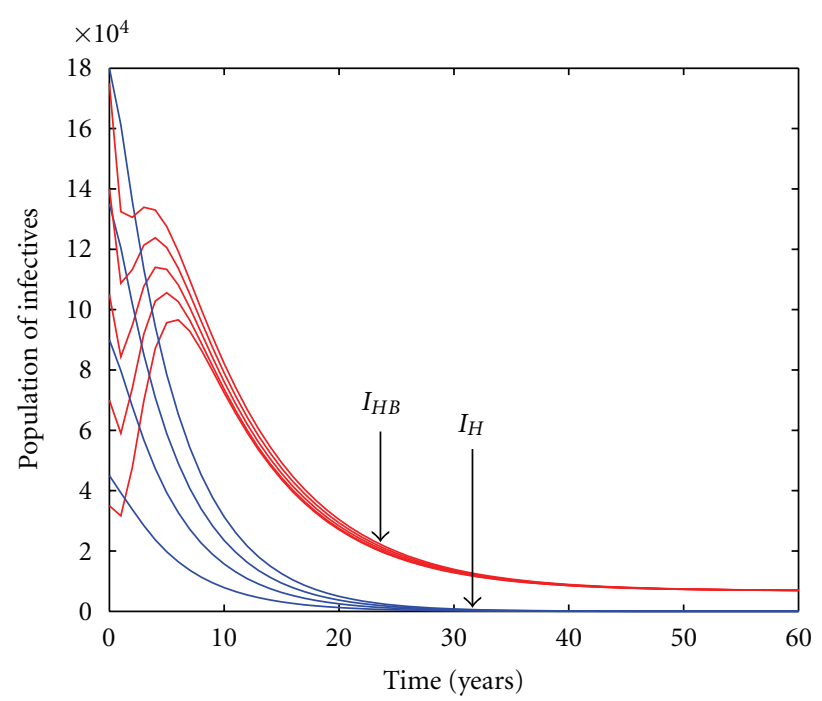

(a)

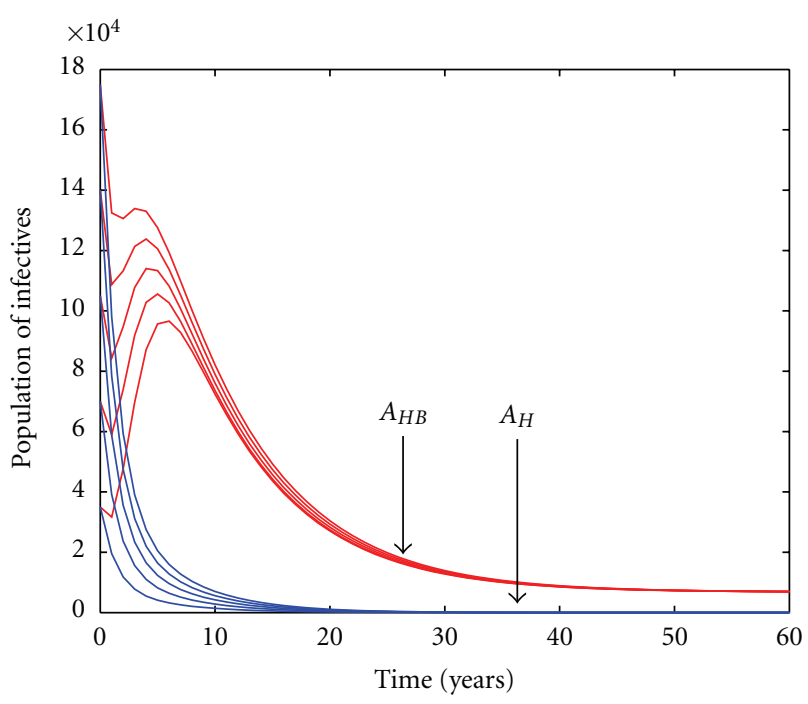

(b)

FIGURE 3: Numerical results of model system (5) showing time series plots of infectives either singly infected with HIV or dually infected with HIV and schistosomiasis for both cases (i.e., either displaying clinical symptoms of AIDS or not), using various initial conditions and parameter values from Table 1.

Figure 3 depicts the effects of schistosomiasis on the dynamics of HIV in the community. The time series plots in Figure 3 suggest that the presence of schistosomiasis in the community might increase the prevalence of HIV/AIDS. These numerical results are in agreement with our analytical results. We note that $I_{H}$ and $A_{H}$ are not reflecting the diseasefree equilibrium, and the convergence is simply due to scale.

\section{Summary and Conclusion}

While schistosomiasis is the second most prevalent neglected tropical disease after hookworm infection (192 million cases worldwide) [5], HIV on the other hand which has killed more than 25 million people since first recognized in 1981 currently affects 33.4 million people, with deaths due to HIV/AIDS-related illnesses standing at about 2 million in 2008 [6]. A mathematical model for investigating the coinfection of schistosomiasis and HIV/AIDS is derived. Comprehensive and qualitative mathematical techniques were used to analyze steady states of the model. The diseasefree equilibrium is shown to be locally asymptotically stable when the associated epidemic threshold known as the basic reproduction number for the model is less than unity. Center manifold theory is used to show that the schistosomiasis-only and HIV/AIDS-only endemic equilibria are locally asymptotically stable when the associated reproduction numbers are greater than unity. The impact of schistosomiasis and its treatment on the dynamics of HIV/AIDS is also investigated. Numerical results are provided to illustrate some of analytical results.

In this study, the impact of schistosomiasis and its treatment on the transmission dynamics of HIV/AIDS in the community is investigated by formulating a mathematical model that incorporates both key epidemiological parameters of both schistosomiasis and HIV/AIDS. Mathematical and numerical analysis of the model suggests that schistosomiasis may increase the prevalence of HIV/AIDS in the community. Analysis of the impact of schistosomiasis treatment has shown that the impact of this form of treatment depends on the sign of a certain threshold parameter $\Theta$, and for $\Theta>1$, schistosomiasis treatment will have a positive impact, for $\Theta=1$, no impact, and for $\Theta<1$, a negative impact on controlling the co-interaction of the two diseases. We, however, note that from schistosomiasis and HIV/AIDS epidemiology, realistic parameter values always yield $1<\Theta$. Consequently, schistosomiasis treatment will always have a positive impact on the control of both schistosomiasis and HIV/AIDS codynamics. Thus, schistosomiasis treatment can reduce the burden of schistosomiasis and HIV/AIDS coinfection in areas of extreme poverty, especially among the rural poor and some disadvantaged urban populations since it is less expensive and usually available in government clinics and hospitals. This outcome highlights the fact that global public health challenges require comprehensive and multipronged approaches to dealing with them. Current efforts that focus on a single infection at a time may be losing substantial rewards of dealing synergistically and concurrently with multiple infectious diseases [7].

\section{Appendix}

In order to establish the conditions for the existence of a bifurcation, we use Theorem 10 proven in [47]. 
Theorem 10. Consider the following general system of ordinary differential equations with a parameter $\phi$ :

$$
\frac{d x}{d t}=f(x, \phi), \quad f: \mathbb{R}^{n} \times \mathbb{R} \longrightarrow \mathbb{R}, f \in \mathbb{C}^{2}\left(\mathbb{R}^{n} \times \mathbb{R}\right),
$$

where 0 is an equilibrium of the system that is $f(0, \phi)=0$ for all $\phi$, and assume that

(A1) $A=D_{x} f(0,0)=\left(\left(\partial f_{i} / \partial x_{j}\right)(0,0)\right)$ is linearization of system (A.1) around the equilibrium 0 with $\phi$ evaluated at 0 . Zero is a simple eigenvalue of $A$, and other eigenvalues of $A$ have negative real parts,

(A2) matrix $A$ has a right eigenvector $u$ and a left eigenvector $v$ corresponding to the zero eigenvalue.

Let $f_{k}$ be the Kth component of $f$ and

$$
\begin{gathered}
a=\sum_{k, i, j=1}^{n} v_{k} u_{i} u_{j} \frac{\partial^{2} f_{k}}{\partial x_{i} \partial x_{j}}(0,0), \\
b=\sum_{k, i=1}^{n} v_{k} u_{i} \frac{\partial^{2} f_{k}}{\partial x_{i} \partial \phi}(0,0) .
\end{gathered}
$$

The local dynamics of (A.1) around 0 are totally governed by a and $b$.

(i) $a>0, b>0$. When $\phi<0$ with $|\phi| \ll 1,0$ is locally asymptotically stable, and there exists a positive unstable equilibrium; when $0<\phi \ll 1,0$ is unstable and there exists a negative and locally asymptotically stable equilibrium.

(ii) $a<0, b<0$. When $\phi<0$ with $|\phi| \ll 1,0$ is unstable and when $0<\phi \ll 1$, asymptotically stable, and there exists a positive unstable equilibrium.

(iii) $a>0, b<0$. When $\phi<0$ with $|\phi| \ll 1,0$ is unstable, and there exists a locally asymptotically stable negative equilibrium; when $0<\phi \ll 1,0$ is stable, and a positive unstable equilibrium appears.

(iv) $a<0, b>0$. When $\phi$ changes from negative to positive, 0 changes its stability from stable to unstable. Correspondingly, a negative equilibrium becomes positive and locally asymptotically stable.

Computations of $a$ and $b$. For system (16), the associated nonzero partial derivatives of $F$ associated with $a$ at the disease-free equilibrium is given by

$$
\begin{aligned}
\frac{\partial^{2} f_{2}}{\partial x_{2} \partial x_{3}} & =\frac{\partial^{2} f_{2}}{\partial x_{3} \partial x_{2}}=-\frac{\beta_{H}^{*} c(1+\eta) \mu_{H}}{\Lambda_{H}}, \\
\frac{\partial^{2} f_{2}}{\partial x_{2}^{2}} & =-\frac{2 \beta_{H}^{*} c \mu_{H}}{\Lambda_{H}}, \\
\frac{\partial^{2} f_{2}}{\partial x_{2} \partial x_{4}} & =\frac{\partial^{2} f_{2}}{\partial x_{4} \partial x_{2}}=-\frac{\beta_{H}^{*} c(1+\kappa) \mu_{H}}{\Lambda_{H}}, \\
\frac{\partial^{2} f_{2}}{\partial x_{3}^{2}} & =-\frac{2 \beta_{H}^{*} c \eta \mu_{H}}{\Lambda_{H}}, \\
\frac{\partial^{2} f_{2}}{\partial x_{3} \partial x_{4}} & =\frac{\partial^{2} f_{2}}{\partial x_{4} \partial x_{3}}=-\frac{\beta_{H}^{*} c(\eta+\kappa) \mu_{H}}{\Lambda_{H}}, \\
\frac{\partial^{2} f_{2}}{\partial x_{4}^{2}} & =-\frac{2 \beta_{H}^{*} c \kappa \mu_{H}}{\Lambda_{H}} .
\end{aligned}
$$

From (A.3), it follows that

$$
a=-\frac{2 \beta_{H}^{*} c \mu_{H} v_{2}}{\Lambda_{H}}\left(u_{2}+u_{3}+u_{4}\right)\left(u_{2}+\eta u_{3}+\kappa u_{4}\right)<0 .
$$

For the sign of $b$, it is associated with the following nonvanishing partial derivatives of $F$ :

$$
\frac{\partial^{2} f_{2}}{\partial x_{2} \partial \beta_{H}^{*}}=c, \quad \frac{\partial^{2} f_{2}}{\partial x_{3} \partial \beta_{H}^{*}}=c \eta, \quad \frac{\partial^{2} f_{2}}{\partial x_{4} \partial \beta_{H}^{*}}=c \kappa,
$$

from which it follows that

$$
b=c\left(u_{2}+\eta u_{3}+\kappa u_{4}\right) v_{2}>0 .
$$

Thus, $a<0$ and $b>0$ and from Theorem 10 item (iv), the result follows.

\section{Acknowledgment}

The authors thank the reviewers for comments and suggestions.

\section{References}

[1] World Health Organization, "The control of schistosomiasis: second report on the WHO expert committee," Tech. Rep., WHO, Geneva, Switzerland, 1993.

[2] http://apps.who.int/tdr/svc/diseases/schistosomiasis.

[3] J. E. Cohen, "Mathematical models of schistosomiasis," Annual Review of Ecology and Systematics, vol. 8, pp. 209-233, 1977.

[4] World Health Organization, The Millennium Development Goals: The Evidence is in: Deworming Helps Meet the Millennium Development Goals, WHO, Geneva, Switzerland, 2005, http://whqlibdoc.who.int/hq/2005/WHO_CDS_CPE_ PVC_2005.12.pdf.

[5] P. J. Hotez and A. Kamath, "Neglected tropical diseases in subSaharan Africa: review of their prevalence, distribution, and disease burden," PLoS Neglected Tropical Diseases, vol. 3, no. 8, article e412, 2009. 
[6] UNAIDS, “AIDS epidemic update, 2009," Joint United Nations Programme on HIV/AIDS (UNAIDS), Geneva, Switzerland, 2009.

[7] L. J. Abu-Raddad, P. Patnaik, and J. G. Kublin, "Dual infection with HIV and malaria fuels the spread of both diseases in SubSaharan Africa," Science, vol. 314, no. 5805, pp. 1603-1606, 2006.

[8] D. Bundy, A. Sher, and E. Michael, "Good worms or bad worms: do worm infections affect the epidemiological patterns of other diseases?" Parasitology Today, vol. 16, no. 7, pp. 273 $312,2000$.

[9] A. M. Elliott, H. Mpairwe, M. A. Quigley et al., "Helminth infection during pregnancy and development of infantile eczema," Journal of the American Medical Association, vol. 294, no. 16, pp. 2032-2034, 2005.

[10] R. M. Maizels, "Infections and allergy—helminths, hygiene and host immune regulation," Current Opinion in Immunology, vol. 17, no. 6, pp. 656-661, 2005.

[11] A. Cooke, P. Tonks, F. M. Jones et al., "Infection with Schistosoma mansoni prevents insulin dependent diabetes mellitus in non-obese diabetic mice," Parasite Immunology, vol. 21, no. 4, pp. 169-176, 1999.

[12] R. W. Summers, D. E. Elliott, J. F. Urban, R. A. Thompson, and J. V. Weinstock, "Trichuris suis therapy for active ulcerative colitis: a randomized controlled trial," Gastroenterology, vol. 128, no. 4, pp. 825-832, 2005.

[13] R. W. Summers, D. E. Elliot, J. F. Urban, R. Thompson, and J. V. Weinstock, "Trichuris suis therapy in Crohn's disease," Gut, vol. 54, no. 1, pp. 87-90, 2005.

[14] J. K. Actor, M. Shirai, M. C. Kullberg, R. M. L. Buller, A. Sher, and J. A. Berzofsky, "Helminth infection results in decreased virus-specific $8+$ cytotoxic $\mathrm{T}$ - cell and Th1 cytokine responses as well as delayed virus clearance," Proceedings of the National Academy of Sciences of the United States of America, vol. 90, no. 3, pp. 948-952, 1993.

[15] M. T. Brady, S. M. O’Neill, J. P. Dalton, and K. H. G. Mills, "Fasciola hepatica suppresses a protective Th1 response against Bordetella pertussis," Infection and Immunity, vol. 67, no. 10, pp. 5372-5378, 1999.

[16] A. L. Chenine, K. A. Buckley, P. L. Li et al., "Schistosoma mansoni infection promotes SHIV clade $\mathrm{C}$ replication in rhesus macaques," AIDS, vol. 19, no. 16, pp. 1793-1797, 2005.

[17] D. Elias, H. Akuffo, C. Thors, A. Pawlowski, and S. Britton, "Low dose chronic Schistosoma mansoni infection increases susceptibility to Mycobacterium bovis BCG infection in mice," Clinical and Experimental Immunology, vol. 139, no. 3, pp. 398-404, 2005.

[18] P. Nunn, D. Kibuga, S. Gathua et al., "Cutaneous hypersensitivity reactions due to thiacetazone in HIV-1 seropositive patients treated for tuberculosis," Lancet, vol. 337, no. 8742, pp. 627-630, 1991.

[19] F. M. Gordin, G. L. Simon, C. B. Wofsy, and J. Mills, “Adverse reactions to trimethoprim-sulfamethoxazole in patients with the acquired immunodeficiency syndrome," Annals of Internal Medicine, vol. 100, no. 4, pp. 495-499, 1984.

[20] E. F. Kjetland, P. D. Ndhlovu, E. Gomo et al., "Association between genital schistosomiasis and HIV in rural Zimbabwean women," AIDS, vol. 20, no. 4, pp. 593-600, 2006.

[21] M. Brown, P. A. Mawa, P. Kaleebu, and A. M. Elliott, "Helminths and HIV infection: epidemiological observations on immunological hypotheses," Parasite Immunology, vol. 28, no. 11 , pp. 613-623, 2006.
[22] M. A. Foulkes, "Advances in HIV/AIDS statistical methodology over the past decade," Statistics in Medicine, vol. 17, no. 1, pp. 1-25, 1998.

[23] C. Castillo-Chavez, "Review of recent models of HIV/AIDS transmission," in Applied Mathematical Ecology, S. Levin, Ed., vol. 18 of Biomathematics Texts, pp. 253-262, Springer, Berlin, Germany, 1989.

[24] H. W Hethcote, Modeling HIV transmission and AIDS in the United States, Lecture Notes in Biomathematics, Springer, New York, NY, USA, 1992.

[25] R. M. May and R. M Anderson, "The transmission dynamics of human immunodeficiency virus (HIV)," in Applied Mathematical Ecology, S. Levin, Ed., vol. 18 of Biomathematics Texts, Springer, 1989.

[26] H. R. Thieme and C. Castillo-Chavez, "How may infectionage-dependent infectivity affect the dynamics of HIV/AIDS?" SIAM Journal on Applied Mathematics, vol. 53, no. 5, pp. 14471479, 1993.

[27] C. Castillo-Chavez, Z. Feng, and D. Xu, "A schistosomiasis model with mating structure and time delay," Mathematical Biosciences, vol. 211, no. 2, pp. 333-341, 2008.

[28] E. J. Allen and H. D. Victory, "Modelling and simulation of a schistosomiasis infection with biological control," Acta Tropica, vol. 87, no. 2, pp. 251-267, 2003.

[29] M. S. Chan and V. S. Isham, "A stochastic model of schistosomiasis immuno-epidemiology," Mathematical Biosciences, vol. 151, no. 2, pp. 179-198, 1998.

[30] Z. Feng, C. C. Li, and F. A. Milner, "Schistosomiasis models with density dependence and age of infection in snail dynamics," Mathematical Biosciences, vol. 177-178, pp. 271286, 2002.

[31] F. A. Milner and R. Zhao, "A deterministic model of schistosomiasis with spatial structure," Mathematical Biosciences and Engineering, vol. 5, no. 3, pp. 505-522, 2008.

[32] R. Zhao and F. A. Milner, "A mathematical model of Schistosoma mansoni in Biomphalaria glabrata with control strategies," Bulletin of Mathematical Biology, vol. 70, no. 7, pp. 1886-1905, 2008.

[33] G. M. Williams, A. C. Sleigh, Y. Li et al., "Mathematical modelling of schistosomiasis japonica: comparison of control strategies in the People's Republic of China," Acta Tropica, vol. 82, no. 2, pp. 253-262, 2002.

[34] P. Zhang, Z. Feng, and F. Milner, "A schistosomiasis model with an age-structure in human hosts and its application to treatment strategies," Mathematical Biosciences, vol. 205, no. 1, pp. 83-107, 2007.

[35] Z. Feng, C. C. Li, and F. A. Milner, "Schistosomiasis models with two migrating human groups," Mathematical and Computer Modelling, vol. 41, no. 11-12, pp. 1213-1230, 2005.

[36] Z. Feng, A. Eppert, F. A. Milner, and D. J. Minchella, "Estimation of parameters governing the transmission dynamics of schistosomes," Applied Mathematics Letters, vol. 17, no. 10, pp. 1105-1112, 2004.

[37] M. E. J. Woolhouse, C. H. Watts, and S. K. Chandiwana, "Heterogeneities in transmission rates and the epidemiology of schistosome infection," Proceedings of the Royal Society B, vol. 245, no. 1313, pp. 109-114, 1991.

[38] J. D. Murray, Mathematical Biology I, An introduction, vol. 17, Springer, Berlin, Germany, 2002.

[39] C. P. Bhunu, J. M. Tchuenche, W. Garira, G. Magombedze, and S. Mushayabasa, "Modeling the effects of schistosomiasis on the transmission dynamics of HIV/AIDS," Journal of Biological Systems, vol. 18, no. 2, pp. 277-297, 2010. 
[40] N. Malunguza, S. Mushayabasa, C. Chiyaka, and Z. Mukandavire, "Modelling the effects of condom use and antiretroviral therapy in controlling HIV/AIDS among heterosexuals, homosexuals and bisexuals," Computational and Mathematical Methods in Medicine, vol. 11, no. 3, pp. 201-222, 2010.

[41] T. C. Porco, J. N. Martin, K. A. Page-Shafer et al., "Decline in HIV infectivity following the introduction of highly active antiretroviral therapy," AIDS, vol. 18, no. 1, pp. 81-88, 2004.

[42] WHO, Guidelines for HIV Diagnosis and Monitoring of Antiretroviral Therapy, WHO, Geneva, Switzerland, 2005.

[43] P. Van den Driessche and J. Watmough, "Reproduction numbers and sub-threshold endemic equilibria for compartmental models of disease transmission," Mathematical Biosciences, vol. 180, pp. 29-48, 2002.

[44] C. P. Bhunu, W. Garira, and G. Magombedze, "Mathematical analysis of a two strain HIV/AIDS model with antiretroviral treatment," Acta Biotheoretica, vol. 57, no. 3, pp. 361-381, 2009.

[45] V. Lakshmikantham, S. Leela, and A. A. Martynyuk, Stability Analysis of Nonlinear Systems, Marcel Dekker, New York, NY, USA, 1989.

[46] J. Carr, Applications of Centre Manifold Theory, Springe, New York, NY, USA, 1981.

[47] C. Castillo-Chavez and B. Song, "Dynamical models of tuberculosis and their applications," Mathematical Biosciences and Engineering, vol. 1, no. 2, pp. 361-404, 2004.

[48] H. Caswell, Matrix Population Models: Construction, Analysis and Interpretation, Sinauer Associates, Sunderland, Mass, USA, 2001.

[49] Nakul Chitnis, James M. Hyman, and Jim M. Cushing, "Determining important parameters in the spread of malaria through the sensitivity analysis of a mathematical model," Bulletin of Mathematical Biology, vol. 70, no. 5, pp. 1272-1296, 2008.

[50] C. Castillo-Chavez, Z. Feng, and W. Huang, "On the computation of $R_{0}$ and its role on global stability," in Mathematical Approaches for Emerging and Reemerging Infectious Diseases: Models, Methods, and Theory, C. Castillo-Chavez et al., Ed., IMA, 126, pp. 215-230, Springer, 2002.

[51] G. Quaittoo, "Bilhazia: Noguchi Closer To Diagnosis," September 2007, http://spectator.newtimesonline.com/. 


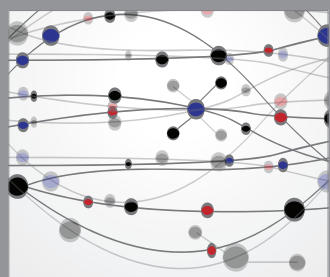

The Scientific World Journal
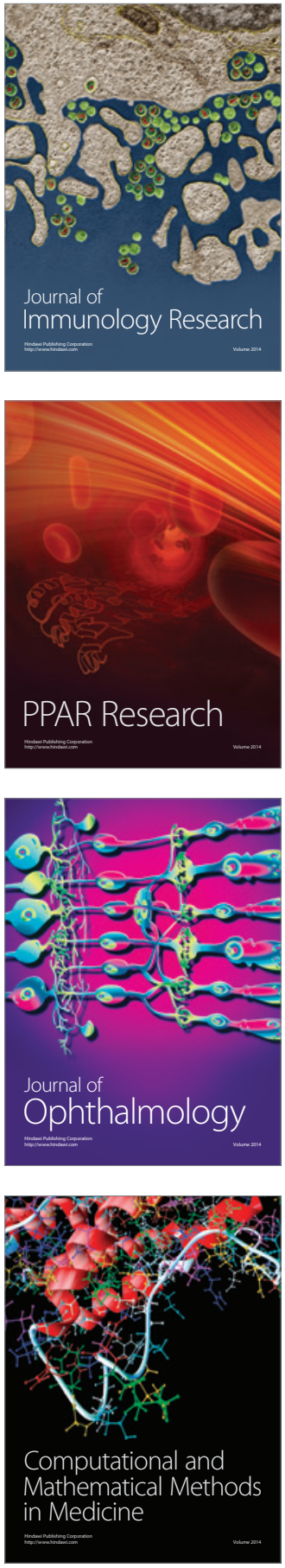

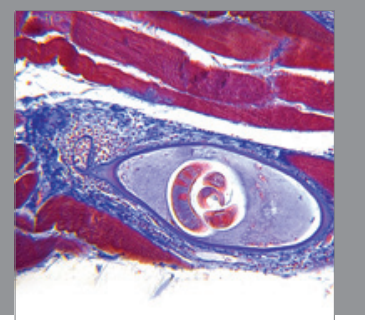

Gastroenterology

Research and Practice
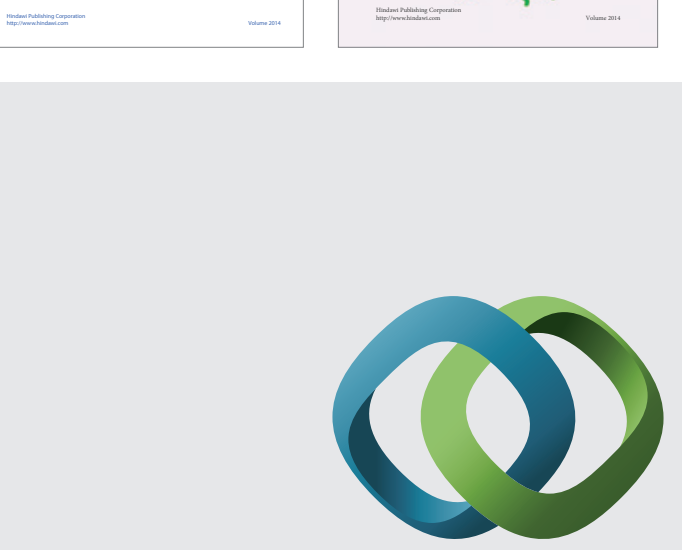

\section{Hindawi}

Submit your manuscripts at

http://www.hindawi.com
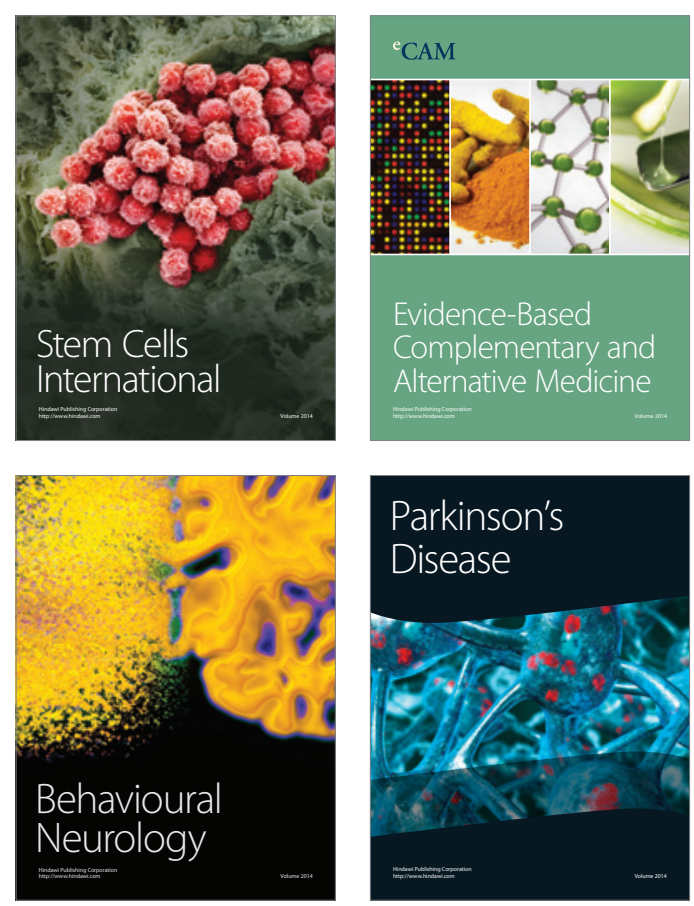

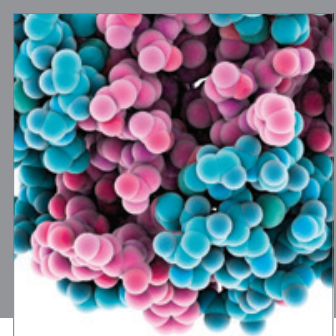

Journal of
Diabetes Research

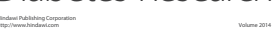

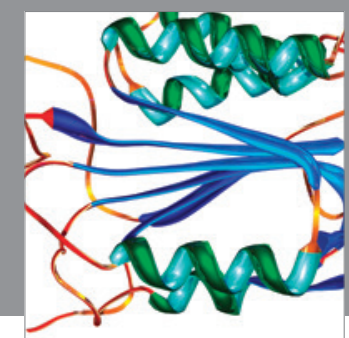

Disease Markers
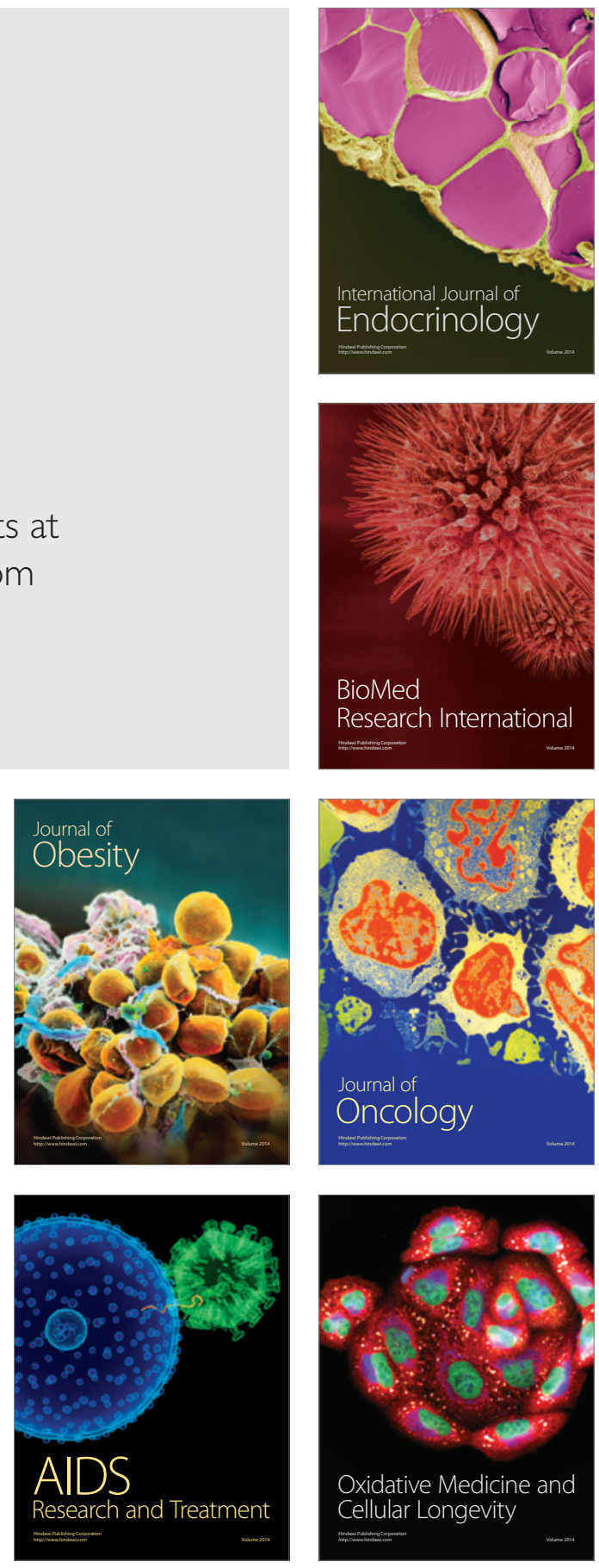